\title{
Nicht-konservative weich strukturvariable Regelungen mit invers-polynomialen Selektionsstrategien
}

\author{
Non-conservative soft variable structure controls with inverse-polynomial selection strategies
}

DOI 10.1515/auto-2015-0033

Eingang 5. März 2015; angenommen 19. November 2015

Zusammenfassung: Der Beitrag stellt notwendige und hinreichende Existenzbedingungen weich strukturvariabler Regelungen mit invers-polynomialen Selektionsstrategien für lineare Strecken mit einer Eingangsgröße und Stellgrößenbeschränkung vor. Das resultierende Regelgesetz wird bezüglich einer Ljapunov-Funktion-basierten unteren Grenze der Konvergenzrate optimiert. Der konvergenzoptimale Regler ist ein Zweipunktregler mit einer einfachen parameterabhängigen Selektionsstrategie. Ein sättigender High-Gain-Regler wird als stetige Approximation dieses Regelgesetzes vorgestellt. Dieser vermeidet die Nachteile des unstetigen Regelgesetzes auf Kosten einer suboptimalen Konvergenzrate.

Schlüsselwörter: Systeme mit einer Eingangsgröße, Stellgrößenbeschränkung, Konvergenzrate, Finsler’s Lemma, Lineare Matrixungleichungen.

\footnotetext{
Abstract: The paper presents necessary and sufficient conditions for the existence of a stabilizing soft variable structure control with inverse-polynomial selection strategies for single-input linear systems with input saturation. The resulting control law is then optimized by maximizing a Lyapunov-Function-based lower bound of the convergence rate. The maximal convergence control is shown to be of bang-bang type, with a simple parameter-dependent switching scheme. To overcome possible difficulties of a switching controller, a saturating high-gain control with

*Korrespondenzautor: Andreea Röthig, Institut für Automatisierungstechnik und Mechatronik, Technische Universität Darmstadt, Darmstadt, E-Mail: aroethig@rmr.tu-darmstadt.de Jürgen Adamy: Institut für Automatisierungstechnik und Mechatronik, Technische Universität Darmstadt, Darmstadt
}

a suboptimal convergence rate, which approximates the bang-bang control, is also presented.

Keywords: Single-input systems, input saturation, convergence-rate, Finsler's Lemma, linear matrix inequalities.

\section{Einleitung}

Eine große Anzahl von Regelungsmethoden beschäftigt sich mit der Stabilisierung linearer zeitinvarianter Systeme mit Stellgrößenbeschränkung. Die lineare Zustandsrückführung ist eine der meist verwendeten Regelgesetze, welche von nichtsättigenden bis zu High-Gain-Regler reicht, wobei auftretende Sättigungseffekte entweder mittels Anti-Windup-Strukturen reduziert [18] oder direkt in der Stabilitätsanalyse [9] berücksichtigt werden. Der generelle Nachteil der linearen Zustandsrückführung ist, dass der Stellgrößenbereich im Bereich kleiner Auslenkungen aufgrund konstanter Regelverstärkung nicht optimal ausgenutzt werden kann und somit oft zu langsamen Ausregelvorgängen führt. Unter den nichtlinearen Regelmethoden erzielt die zeitoptimale Regelung, in diesem Fall ein Zweipunktregler, den schnellsten Ausregelvorgang. Da die Umschaltstrategie des zeitoptimalen Reglers aber generell nicht analytisch vorgebbar ist, wird diese Methode oft nur bei Systemen niedrigerer Ordnung verwendet. Außerdem kann die Diskontinuität des Regelgesetzes in der Praxis technische Probleme verursachen. Beispielsweise kann die ununterbrochene Aktivität des Reglers aufgrund unvermeidbaren Rauschens die Aktoren beschädigen. Eine Alternative zu den beiden Regelungsmethoden bieten die strukturvariablen Regelungen ohne Gleitzustand, welche durch variable Verstärkungen den Nachteil der linearen Zustandsrückführung überwinden und durch einfache Entwurfsverfahren bei Systemen beliebiger Ordnung verwendet werden können. Im 
Falle der strukturvariablen Regelung ohne Gleitzustand wird zwischen verschiedenen Regelgesetzen während des Ausregelvorgangs umgeschaltet. Dabei unterscheidet man zwischen parameter- und strukturumschaltenden Regelgesetzen. In dieser Arbeit wird eine Unterklasse der Ersteren betrachtet. Eine Übersicht solcher Regelungen bietet [1]. Die erste systematisch entwickelte Regelung dieser Art findet sich in [13]. Erfolgt die Parameteränderung kontinuierlich während des Ausregelvorgangs, so spricht man von weich strukturvariabler Regelung (WSVR). Bei der hier analysierten Klasse von WSVR ergibt sich der Parameterwert aus einer Selektionsstrategie, die das Einzugsgebiet der Ruhelage in infinitesimal dicht ineinander verschachtelte Gebiete unterteilt. Dem Rand jedes Gebiets wird ein eindeutiger Wert des Parameters zugewiesen. Die Ellipsoide sind darüber hinaus durch Inverse von polynomialen Matrizen definiert. Eine ähnliche Klasse von WSVR wurde in [12] eingeführt. Darin wurden hinreichende Stabilisierbarkeitsbedingungen linearer Systeme mit Stellgrößenbeschränkung durch WSVR mittels impliziter Ljapunov-Funktionen (iLF) und polynomialer Selektionsstrategien vorgestellt.

Die Geschwindigkeit des Ausregelvorgangs wird oft mit der Konvergenzrate exponentiell stabiler Systeme in Verbindung gebracht, welche als kleinster Abklingfaktor definiert ist, mit der die Norm eines Zustands zu null strebt. Für stabile lineare zeitinvariante Systeme (LTI) ist die Konvergenzrate konstant im gesamten Zustandsraum und entspricht dem Betrag des Realteils des Eigenwertes, der am nächsten zur Imaginärachse liegt. Für nichtlineare Systeme hängt sie von dem Abstand zur Ruhelage ab. In der Literatur wird sie daher mit Hilfe von kontraktiv invarianten Gebieten analysiert, vgl. z.B. [5]. Diese zeichnen sich dadurch aus, dass Trajektorien, die in solche Gebiete eintreten, diese nicht mehr verlassen. Dabei sind die meist verwendeten kontraktiv invarianten Gebiete ellipsoidaler Form, da diese mit effizienten Hilfsmitteln, wie der Ljapunov Gleichung und, generell, der linearen Matrixungleichungen (LMI) analysiert werden können. Die Vorteile der ellipsoidalen Gebiete werden auch im Fall strukturvariabler Regelungen genutzt, indem die Variation der Reglerverstärkungen mit solchen Gebieten im Zustandsraum verbunden wird.

Das Regelgesetz, das mit Hilfe quadratischer Ljapunov-Funktionen $V(\mathbf{x})=\mathbf{x}^{\top} \mathbf{P} \mathbf{x}$ eine untere Grenze der Konvergenzrate maximiert, ist ein Zweipunktregler mit einer einfachen Umschaltstrategie, vgl. z.B. [9]. Das Regelgesetz hat die Form $u=-\operatorname{sgn}\left(\mathbf{b}^{\top} \mathbf{P x}\right)$, wobei $\mathbf{b}$ der Eingangsvektor der Strecke ist. Die maximale Konvergenzrate hängt von der Matrix $\mathbf{P}$ ab, welche auch ein kontraktiv invariantes Ellipsoid determiniert. In [9] wurde gezeigt, dass die Größe des erzielten Ellipsoids im Konflikt mit der Höhe der Konvergenzrate steht, d.h. eine hohe Konvergenzrate erzielt ein kleines Ellipsoid und umgekehrt. Durch die Verwendung einer parameterabhängigen Matrix $\mathbf{P}_{v}$, wie im Falle strukturvariabler Regelungen, besteht die Möglichkeit, die Größe des Ellipsoids an den Abstand zur Ruhelage anzupassen und somit die Konvergenzrate insgesamt zu verbessern. Vgl. auch [7] für eine solche Verbesserung durch eine parameterabhängige Matrix. Darin ist die Selektionsstrategie ebenfalls durch eine inverse polynomiale Matrix definiert, jedoch ist der Grad des Polynoms auf eins beschränkt.

In diesem Beitrag wird eine Regelmethode mit inverspolynomialen Selektionsstrategien vorgestellt, wobei der Grad des Polynoms beliebig gewählt werden kann. Um die Vorteile der LMIs weiterhin ausnutzen zu können, werden Bedingungen angegeben, die für die Stabilisierbarkeit eines linearen Systems mit einer Eingangsgröße und Stellgrößenbeschränkung nicht nur hinreichend sondern auch notwendig sind. Die Bedingungen bauen auf die notwendigen und hinreichenden Bedingungen für die Existenz eines linearen Zustandsreglers auf, welche in [11] vorgestellt wurden. Durch eine konstruktive Methode werden somit nicht-konservative Existenzbedingungen einer WSVR mittels invers-polynomialen Selektionsstrategien vorgestellt. Sind die Bedingungen erfüllt, so wird ein beschränkter Regler angegeben, der das System stabilisiert.

Die dabei erzielten invarianten Gebiete werden anschließend zur Optimierung einer Ljapunov-Funktionbasierten unteren Grenze der Konvergenzrate verwendet. Es wird gezeigt, dass die Optimierung dieser Konvergenzrate einen Zweipunktregler mit einer parameterabhängigen Umschaltstrategie erzielt. Um die Nachteile der Diskontinuität des Regelgesetzes zu umgehen, wird eine stetige Approximation des konvergenzoptimalen Regelgesetzes vorgestellt, die auf Kosten einer leichten Verschlechterung der Konvergenzrate einen stetigen Stellgrößenverlauf erzielt. Dabei wird auch ein nichtkonservativer Entwurf des konvergenzoptimalen Regelgesetzes vorgestellt. Dieser beinhaltet Stabilitätsbedingungen, welche sowohl notwendig als auch hinreichend für die Stabilisierbarkeit einer linearen Strecke mit Stellgrößenbeschränkung durch eine WSVR dieser Klasse sind.

Der Beitrag ist wie folgt gegliedert: Abschnitte 1.1 und 1.2 enthalten die verwendeten Notationen und Definitionen, sowie einen Satz aus [2], der für die Stabilitätsuntersuchung der vorgestellten Regler notwendig ist. Abschnitt 2 zeigt die notwendigen und hinreichenden Bedingungen für die Stabilisierbarkeit linearer Strecken mit Stellgrößenbeschränkung durch WSVR mit inverspolynomialen Selektionsstrategien. Abschnitt 3 zeigt die 
notwendigen und hinreichenden Existenzbedingungen der konvergenzoptimalen WSVR, sowie die des stetig approximierenden Regelgesetzes. Abschließend stellen Abschnitt 4 die Entwurfsschritte und die Abschnitte 5 und 6 zwei Entwurfsbeispiele dar. In Abschnitt 7 werden die Ergebnisse zusammengefasst und es wird ein Ausblick gegeben.

\subsection{Vorbemerkungen}

Folgende Notationen werden verwendet:

\begin{tabular}{|c|c|}
\hline$:=$ & definiert als \\
\hline $\mathbb{R}_{*}^{n}$ & $\mathbb{R}^{n} \backslash\{\mathbf{0}\}$ \\
\hline $\mathbb{P}^{n \times n}$ & $n \times n$ positiv definite Matrizen \\
\hline $\operatorname{Sym}^{n}$ & $n \times n$ symmetrische Matrizen \\
\hline Skew $^{n}$ & $n \times n$ schiefsymmetrische Matrizen \\
\hline$\partial_{t} g(\mathbf{x}(t), v)$ & $\Leftrightarrow \frac{\partial g}{\partial x} \cdot \dot{\mathbf{x}}$ \\
\hline$\partial_{v} g(\mathbf{x}, v)$ & $\Leftrightarrow \partial g(\mathbf{x}, v) / \partial v$ \\
\hline$\|\mathbf{x}\|$ & Euklidische Norm eines Vektors \\
\hline $\mathbf{x}^{+}$ & Pseudoinverse eines Vektors $\mathbf{x} \in \mathbb{R}^{n}$, \\
\hline $\mathbf{v}^{[k]}$ & {$\left[\begin{array}{llll}1 & v & \cdots & v^{k-1}\end{array}\right]^{\top}$} \\
\hline$A>0$ & $\mathbf{A} \in \operatorname{Sym}^{n}$, mit $\mathbf{x}^{\top} \mathbf{A} \mathbf{x}>0, \forall \mathbf{x} \neq \mathbf{0}$ \\
\hline $\mathbf{A}>\mathbf{B}$ & $\Leftrightarrow \mathbf{A}-\mathbf{B} \in \mathbb{P}^{n \times n}$ \\
\hline $\operatorname{Spec}(\mathbf{A})$ & Spektrum einer Matrix $\mathbf{A} \in \mathbb{R}^{n \times n}$ \\
\hline$\lambda_{i}(\mathbf{A})$ & $\begin{array}{l}\text { i. Eigenwert einer Matrix } \mathbf{A} \text {, mit } \operatorname{Spec}(\mathbf{A}) \subset \mathbb{R} \\
\text { und } \lambda_{1}(\mathbf{A})>\lambda_{n}(\mathbf{A})\end{array}$ \\
\hline $\mathbf{P}^{A}$ & die Adjunkte einer Matrix $\mathbf{P} \in \mathbb{R}^{n \times n}$ \\
\hline $\mathbf{P}_{v}$ & von $v$ abhängige Matrix $\mathbf{P}(v)$ \\
\hline $\mathcal{G}_{\star}(v)$ & Gebiet $\left\{\mathbf{x} \in \mathbb{R}^{n} \mid g_{\star}(\mathbf{x}, v)<0\right\}$ \\
\hline$\varepsilon$ & Ellipsoid $\left\{\mathbf{x} \in \mathbb{R}^{n} \mid \mathbf{x}^{\top} \mathbf{P} \mathbf{x}<1\right\}$ \\
\hline $\mathcal{E}(v)$ & Ellipsoid $\left\{\mathbf{x} \in \mathbb{R}^{n} \mid \mathbf{x}^{\top} \mathbf{P}(v) \mathbf{x}<1\right\}$ \\
\hline$\partial \mathcal{G}_{\star}$ & Rand eines Gebiets $\mathcal{G}_{\star}$ \\
\hline $\mathcal{N}(\mathbf{A})$ & Kern (Nullraum) von $\hat{A} \in \mathbb{R}^{m \times n}$ \\
\hline $\operatorname{dim}(\mathcal{N}(\mathbf{A}))$ & $\begin{array}{l}n-\operatorname{Rang}\left(\mathbf{A}^{\top}\right), \text { mit } \mathbf{A} \in \mathbb{R}^{m \times n}, \mathcal{N}(\mathbf{A}):=\{\mathbf{x} \in \\
\left.\mathbb{R}^{n} \mid \mathbf{A x}=\mathbf{0}\right\}\end{array}$ \\
\hline $\mathcal{B}_{\delta}(\mathbf{0})$ & $\left\{\mathbf{x} \in \mathbb{R}^{n} \mid\|\mathbf{x}\|<\delta\right\}$ \\
\hline $\mathcal{H}(\varepsilon)$ & $\begin{array}{l}\left\{(\mathbf{x}, v) \mid \mathbf{x} \in \mathcal{U}_{0} \backslash\{\mathbf{0}\}, \varepsilon<v<1\right\} \text { wobei } \mathcal{U}_{0} \subseteq \\
\mathcal{B}_{\delta}(\mathbf{0})\end{array}$ \\
\hline
\end{tabular}

Folgende Definitionen werden verwendet:

Definition 1 (Kontraktiv invariantes Ellipsoid). Für eine gegebene positiv definite Matrix $\mathbf{P}>\mathbf{0}$ heißt ein Ellipsoid $\mathcal{E}:=\left\{\mathbf{x} \in \mathbb{R}^{n} \mid \mathbf{x}^{\top} \mathbf{P} \mathbf{x}<1\right\}$ kontraktiv invariant für ein System $\dot{\mathbf{x}}=\mathbf{f}(\mathbf{x})$, wenn für $V(\mathbf{x}):=\mathbf{x}^{\top} \mathbf{P} \mathbf{x}, \dot{V}(\mathbf{x})=2 \mathbf{x}^{\top} \mathbf{P} \mathbf{f}(\mathbf{x})<0$, $\forall \mathbf{x} \in \mathcal{E} \backslash\{\mathbf{0}\}$ gilt. $^{1}$

1 In diesem Fall konvergieren alle Trajektorien, die in $\varepsilon$ starten, asymptotisch gegen die Ruhelage $\mathbf{x}_{\mathrm{R}}=\mathbf{0}$.
Definition 2 (Verschachtelte Gebiete). Zwei Gebiete $\mathcal{G}\left(v_{1}\right)$ und $\mathcal{G}\left(v_{2}\right)$, mit $0<v_{2}<v_{1} \leq \bar{v}$, heißen verschachtelt im Intervall $v \in(0, \bar{v}]$, wenn deren Ränder keine gemeinsamen Punkte haben, d. h. wenn $\partial \mathcal{G}\left(v_{1}\right) \cap \partial \mathcal{G}\left(v_{2}\right)=\emptyset$, und wenn $\mathcal{G}\left(v_{2}\right) \subset \mathcal{G}\left(v_{1}\right)$ gilt.

Definition 3 (Voll-Rang-Faktorisierung einer Matrix). Gegeben sei eine Matrix $\mathbf{A} \in \mathbb{R}^{m \times n}$, mit $\operatorname{Rang}(\mathbf{A})=r$. Das Matrizentupel $\left(\mathbf{A}_{l}, \mathbf{A}_{r}\right)$, mit $\mathbf{A}_{l} \in \mathbb{R}^{m \times r}, \operatorname{Rang}\left(\mathbf{A}_{l}\right)=r$ und $\mathbf{A}_{r} \in \mathbb{R}^{r \times n}, \operatorname{Rang}\left(\mathbf{A}_{r}\right)=r$ heißt eine Voll-RangFaktorisierung der Matrix A wenn $\mathbf{A}=\mathbf{A}_{l} \mathbf{A}_{r}$.

Folgender Satz aus [2] stellt hinreichende Bedingungen für die asymptotische Stabilität der Ruhelage eines nichtlinearen Systems mittels impliziter Ljapunov-Funktionen dar. Der Satz verknüpft die direkte Methode von Ljapunov mit dem Satz über implizite Funktionen².

Satz 1. Gegeben sei die stetige Funktion $\mathbf{h}(\mathbf{x})$ und das System

$$
\dot{\mathbf{x}}=\mathbf{h}(\mathbf{x}), \quad \mathbf{x} \in \mathbb{R}^{n},
$$

mit der Ruhelage $\mathbf{x}_{\mathrm{R}}=\mathbf{0}$ und eindeutiger Lösung für jeden Anfangswert. Wenn eine stetige und differenzierbare Funktion $g(\mathbf{x}, v): \mathcal{H}(0) \rightarrow \mathbb{R}$ existiert, welche die Bedingungen

(i) Aus $g(\mathbf{x}, v)=0$, folgt: $\mathbf{x}=\mathbf{0} \Leftrightarrow v \rightarrow 0^{+}$,

(ii) $\lim _{v \rightarrow 0^{+}} g(\mathbf{x}, v)>0$ und

$$
\lim _{v \rightarrow 1^{-}} g(\mathbf{x}, v)<0, \forall \mathbf{x} \in \mathcal{U}_{0} \backslash\{\mathbf{0}\}
$$

(iii) $\quad-\infty<\partial_{v} g(\mathbf{x}, v)<0, \quad \forall(\mathbf{x}, v) \in \mathcal{H}(0)$,

(iv) $\partial_{t} g(\mathbf{x}(t), v)<0, \quad \forall(\mathbf{x}, v) \in \mathcal{H}(0)$,

erfüllt, dann ist die Ruhelage $\mathbf{x}_{\mathrm{R}}=\mathbf{0}$ asymptotisch stabil, und die Gebiete

$$
\mathcal{G}(v):=\left\{\mathbf{x} \in \mathbb{R}^{n} \mid g(\mathbf{x}, v)<0\right\} \subseteq \mathcal{U}_{0}
$$

sind verschachtelt und kontraktiv invariant für alle $v \in$ $(0,1)$.

Beweis. Der Beweis des Satzes bezüglich der asymptotischen Stabilität der Ruhelage kann in [2, Satz 4] gefunden werden. Die kontraktive Invarianz der Gebiete $\mathcal{G}(v)$ wird in [2, Satz 5] und deren Verschachtelung in [2, Abschnitt III] nachgewiesen. Im Folgenden wird nur die Beweisidee für die asymptotische Stabilität der Ruhelage skizziert.

Die Bedingungen (ii) und (iii) stellen sicher, dass die Selektionsstrategie $g(\mathbf{x}, v)=0$ eine eindeutige Lösung für

2 Vgl. [19]. 
jedes $\mathbf{x} \in \mathcal{U}_{0} \backslash\{\mathbf{0}\}$ hat, ${ }^{3}$ welche eine stetige Funktion $v=$ $v(\mathbf{x})$ ist. Darüber hinaus stellt die Funktion $0<v(\mathbf{x})<1$, welche durch $g(\mathbf{x}, v)=0$ implizit definiert ist, und - aufgrund der Bedingung $(i)$ - für $\mathbf{x}=\mathbf{0}$ stetig erweiterbar mit $v(\mathbf{0})=0$ ist, eine implizite Ljapunov-Funktion des Systems dar. Dies resultiert aus der Tatsache, dass $v(\mathbf{x})>0$ und $\dot{v}(\mathbf{x})<0$, da aufgrund der Bedingungen (iii) und (iv) gilt

$$
\dot{v}(\mathbf{x}(t))=-\frac{\partial_{t} g(\mathbf{x}(t), v)}{\partial_{v} g(\mathbf{x}, v)}<0, \quad \forall(\mathbf{x}, v) \in \mathcal{H}(0) .
$$

Korollar 2 ([2]). Alle Trajektorien, die auf dem Rand eines Gebietes $\mathcal{G}(v)$ starten $-\mathrm{d}$. h. für $\mathbf{x}(0) \in \partial \mathcal{G}(v)$, mit $v \in(0,1)$ - laufen für $t>0$ in das Gebiet hinein.

Beweis. Dies folgt unmittelbar aus Bedingung (iv) des Satzes 1 , da für ein beliebiges $\Delta t>0$ gilt $g(\mathbf{x}(t+\Delta t), v)<$ $g(\mathbf{x}(t), v)=0, \forall \mathbf{x} \in \partial \mathcal{G}(v), v \in(0,1)$. Der Zustand $\mathbf{x}(t+\Delta t)$ wird in das Ellipsoid $\mathcal{G}(v)$ hineinlaufen.

\subsection{Definition einer stabilisierenden WSVR mit invers-polynomialen Selektionsstrategien}

Im Folgenden betrachten wir LTI-Systeme gegeben durch

$$
\dot{\mathbf{x}}=\mathbf{A x}+\mathbf{b} u, \quad \mathbf{x} \in \mathbb{R}^{n}, \quad u \in \mathbb{R},|u| \leq 1 .
$$

Die allgemeine Form der hier analysierten WSVR mit invers-polynomialen Selektionsstrategie ist

$$
u=-f(\mathbf{x}, v)
$$

wobei der Parameter $v \in[\varepsilon, 1]$, mit $\varepsilon \in(0,1)$, für ein gegebenes $\mathbf{x} \in \mathbb{R}_{*}^{n}$ durch eine Gleichung

$$
g_{\mathrm{u}}(\mathbf{x}, v):=\mathbf{x}^{\top} \mathbf{R}_{v}^{-1} \mathbf{x}-1=0, \quad \mathbf{R}_{v}:=\sum_{i=\mathrm{M}_{\mathrm{L}}}^{\mathrm{M}_{\mathrm{U}}} v^{i} \mathbf{R}_{\mathrm{c}_{i}},
$$

mit $\mathbf{R}_{\mathrm{c}_{i}} \in \operatorname{Sym}^{n}, \quad i=\mathrm{M}_{\mathrm{L}}, \ldots, \mathrm{M}_{\mathrm{U}}, \mathrm{M}_{\mathrm{L}}, \mathrm{M}_{\mathrm{U}} \in \mathbb{Z}, \mathrm{M}_{\mathrm{L}}<$ $0, \mathrm{M}_{\mathrm{L}} \leq \mathrm{M}_{\mathrm{U}}$ und $\mathbf{R}_{v}>\mathbf{0}, \forall v \in[\varepsilon, 1]$, bestimmt wird. Dabei ist $\mathbf{R}_{v}$ eine polynomiale Matrix. ${ }^{4}$

3 Vgl. [2, Satz 3]. Die Interpretation dieser Bedingungen lautet wie folgt. Bedingung ii) stellt sicher, dass im Falle jedes festen $\mathbf{x} \in \mathcal{U}_{0} \backslash\{\mathbf{0}\}$ für jedes hinreichend kleine $\epsilon>0 g(\mathbf{x}, \epsilon)>0$ und $g(\mathbf{x}, 1-\epsilon)<0$ gelten. Da die Funktion $g(\mathbf{x}, v)$ auch stetig in $\mathcal{H}(0)$ ist, weist diese mindestens eine Nullstelle für das jeweilige $\mathbf{x} \in \mathcal{U}_{0} \backslash\{\mathbf{0}\}$ auf. Aufgrund der strengen Monotonie dieser Funktion aus Bedingung iii) folgt darüber hinaus, dass die Nullstelle eindeutig ist.

4 Strenggenommen handelt es sich um Laurent-Polynome, welche auch negative Exponenten zulassen. Diese Unterscheidung spielt jedoch im Weiteren keine Rolle.
Das Regelgesetz aus Gleichungen (3)-(4) für das System aus Gleichung (2) heißt stabilisierende WSVR mit invers-polynomialer Selektionsstrategie, wenn die Gebiete

$$
\mathcal{E}_{\mathrm{u}}(v):=\left\{\mathbf{x} \in \mathbb{R}^{n} \mid g_{\mathrm{u}}(\mathbf{x}, v)=\mathbf{x}^{\top} \mathbf{R}_{v}^{-1} \mathbf{x}-1<0\right\}
$$

für alle $v \in[\varepsilon, 1]$ verschachtelt und kontraktiv invariant sind, und $|u| \leq 1, \forall v \in[\varepsilon, 1]$ gilt.

\section{Notwendige und hinreichende Existenzbedingungen einer stabilisierenden WSVR mit invers-polynomialen Selektionsstrategien}

Der folgende Satz mitsamt einer konstruktiven Beweismethode stellt die notwendigen und hinreichenden Bedingungen für die Existenz einer stabilisierenden WSVR mit invers-polynomialen Selektionsstrategien dar. Für eine beliebige lineare Strecke mit einer Eingangsgröße ergibt sich daraus (im Existenzfall) ein beschränktes stabilisierendes Regelgesetz. Dieses kann verwendet werden, wenn eine beliebige stabilisierende Regelung für die Initialisierung eines Optimierungsalgorithmus gesucht wird.

Satz 3. Gegeben sei das LTI-System aus Gleichung (2) und eine reelle Zahl $\varepsilon \in(0,1)$. Folgende Aussagen sind äquivalent:

i) Es existieren die ganzen Zahlen $\mathrm{M}_{\mathrm{L}}, \mathrm{M}_{\mathrm{U}} \in \mathbb{Z}$, mit $\mathrm{M}_{\mathrm{L}}<0$, $\mathrm{M}_{\mathrm{L}} \leq \mathrm{M}_{\mathrm{U}}$, die Matrizen $\mathbf{R}_{\mathrm{c}_{i}} \in$ Sym ${ }^{n}$, mit $i=\mathrm{M}_{\mathrm{L}}, \ldots, \mathrm{M}_{\mathrm{U}}$, sowie eine rationale Vektorfunktion $\mathbf{k}_{v}:[\varepsilon, 1] \rightarrow \mathbb{R}^{n}$, sodass für alle $v \in[\varepsilon, 1]$ die ellipsoidalen Gebiete

$$
\mathcal{E}_{\mathrm{P}}(v):=\left\{\mathbf{x} \in \mathbb{R}^{n} \mid g_{\mathrm{P}}(\mathbf{x}, v)<0\right\},
$$

mit

$$
\begin{aligned}
g_{\mathrm{P}}(\mathbf{x}, v) & :=\mathbf{x}^{\top} \mathbf{Q}_{v} \mathbf{x}-1, \\
\mathbf{Q}_{v} & :=\mathbf{R}_{v}^{-1}=\frac{1}{\operatorname{det}\left(\mathbf{R}_{v}\right)} \mathbf{R}_{v}^{A}, \\
\mathbf{R}_{v} & :=\sum_{i=\mathrm{M}_{\mathrm{L}}}^{\mathrm{M}_{\mathrm{U}}} v^{i} \mathbf{R}_{\mathrm{c}_{i}}>\mathbf{0}, \quad \forall v \in[\varepsilon, 1],
\end{aligned}
$$

für das System aus Gleichung (2) mit einem Regelgesetz

$$
u=-\mathbf{k}_{v}^{\top} \mathbf{x}, \quad \mathbf{k}_{v}:[\varepsilon, 1] \rightarrow \mathbb{R}^{n},
$$

verschachtelt und kontraktiv invariant sind, und

$$
|u| \leq 1, \quad \forall \mathbf{x} \in \mathcal{E}_{\mathrm{p}}(v), v \in[\varepsilon, 1],
$$

gilt. 
ii) Es existieren die ganzen Zahlen $\mathrm{m}_{1}, \mathrm{~m}_{\mathrm{u}}$, mit $\mathrm{m}_{1}<0$ und $\mathrm{m}_{\mathrm{l}} \leq \mathrm{m}_{\mathrm{u}}$, sowie die Matrizen $\mathbf{P}_{\mathrm{c}_{i}} \in \operatorname{Sym}^{n}, i=\mathrm{m}_{\mathrm{l}}, \ldots, \mathrm{m}_{\mathrm{u}}$, sodass für jedes $v \in[\varepsilon, 1]$ gilt

$$
\begin{gathered}
\mathbf{P}_{v}=\sum_{i=\mathrm{m}_{1}}^{\mathrm{m}_{u}} v^{i} \mathbf{P}_{c_{i}}>\mathbf{0}, \\
\partial_{v} \mathbf{P}_{v}=\sum_{i=\mathrm{m}_{1}}^{\mathrm{m}_{\mathrm{u}}} i v^{i-1} \mathbf{P}_{c_{i}}>\mathbf{0}, \\
\mathbf{A P}_{v}+\mathbf{P}_{v} \mathbf{A}^{\top} \prec \mathbf{b} \mathbf{b}^{\top} .
\end{gathered}
$$

Korollar 4. Falls ii) gilt, dann ist ein stabilisierendes Regelgesetz in $i$ ) gegeben durch

$$
\mathbf{k}_{v}^{\top}=r \mathbf{b}^{\top} \mathbf{P}_{v}^{-1}, \quad \mathbf{R}_{v}=d \mathbf{P}_{v},
$$

sowie

$$
\frac{1}{d \cdot r^{2}} \geq \mathbf{b}^{\top} \mathbf{P}_{\varepsilon}^{-1} \mathbf{b}>0, \quad r \geq \frac{1}{2} .
$$

Bemerkung 1. Die parameterabhängigen Bedingungen aus Gleichungen (10)-(12) stellen polynomiale Matrizen in $v \in[\varepsilon, 1]$ dar und können in äquivalente parameterunabhängige LMIs transformiert werden. Die Transformation wurde in [20] vorgestellt. Sie beruht auf der verallgemeinerten $S$-Prozedur aus [10]. In [12, Anhang A.4] werden auch einfache Matlab-Funktionen zur Verfügung gestellt, womit man solche Bedingungen in äquivalente parameterunabhängige LMIs transformieren kann.

Bemerkung 2. Der Übersichtlichkeit halber wird eine Skizze des Beweises vorgestellt. Mit Hilfe des Satzes 1 aus [2] wird erstens bewiesen, dass die Bedingungen (10)(12), unter Verwendung des Regelgesetzes aus Gleichungen (8), (13)-(14), hinreichend dafür sind, dass die ellipsoidalen Gebiete $\mathcal{G}_{\mathrm{p}}(v)$ aus Gleichung (6) verschachtelt und kontraktiv invariant sind. Die Notwendigkeit der Bedingungen (10)-(12) wird anschließend mit Hilfe des Finsler's Lemmas, vgl. z.B. [16], gezeigt.

Beweis. ii) $\Rightarrow$ i) Für den Beweis wird die Notation $\mathbf{A}_{v}:=$ $\mathbf{A}-r \mathbf{b} \mathbf{b}^{\top} \mathbf{P}_{v}^{-1}$ verwendet. Aus Gleichung (12) folgt, dass

$$
\begin{aligned}
\mathbf{A}_{v} \mathbf{P}_{v} & +\mathbf{P}_{v} \mathbf{A}_{v}^{\top}=\mathbf{A P}_{v}+\mathbf{P}_{v} \mathbf{A}^{\top}-2 r \mathbf{b} \mathbf{b}^{\top} \\
& \prec \mathbf{A P}_{v}+\mathbf{P}_{v} \mathbf{A}^{\top}-\mathbf{b} \mathbf{b}^{\top} \prec \mathbf{0}, \quad \forall v \in[\varepsilon, 1],
\end{aligned}
$$

da $r \geq 0.5$ ist. Durch Links- und Rechtsmultiplizieren der Gleichung (15) mit der positiv definiten Matrix $\mathbf{P}_{v}^{-1}$ ergibt sich

$$
\mathbf{P}_{v}^{-1} \mathbf{A}_{v}+\mathbf{A}_{v}^{\top} \mathbf{P}_{v}^{-1} \prec \mathbf{0}, \quad \forall v \in[\varepsilon, 1]
$$

Aus $\mathbf{Q}_{v}=d^{-1} \mathbf{P}_{v}^{-1}$ folgt, dass eine Umgebung $\mathcal{U}_{0}$ der Ruhelage existiert, sodass für die Ableitung der Funktion
$g_{\mathrm{P}}(\mathbf{x}, v)$ aus Gleichung (7) entlang der Trajektorien des Systems $\dot{\mathbf{x}}=\mathbf{A}_{v} \mathbf{x}$

$$
\partial_{t} g_{\mathrm{P}}(\mathbf{x}(t), v)=\mathbf{x}^{\top}\left(\mathbf{A}_{v}^{\top} \mathbf{Q}_{v}+\mathbf{Q}_{v} \mathbf{A}_{v}\right) \mathbf{x}<0,
$$

$\forall(\mathbf{x}, v) \in \mathcal{H}(\varepsilon)$ gilt. Daraus folgt, dass die Bedingung (iv) des Satzes 1 für alle $(\mathbf{x}, v) \in \mathcal{H}(\varepsilon)$ erfüllt ist. Darüber hinaus gilt

$$
\begin{aligned}
\partial_{v} \mathbf{Q}_{v} & =\partial_{v}\left(d^{-1} \mathbf{P}_{v}^{-1}\right) \\
& =-d^{-1} \mathbf{P}_{v}^{-1}\left(\partial_{v} \mathbf{P}_{v}\right) \mathbf{P}_{v}^{-1} \prec \mathbf{0}, \quad \forall v \in[\varepsilon, 1],
\end{aligned}
$$

d.h.

$$
-\infty<\partial_{v} g_{\mathrm{P}}(\mathbf{x}, v)=\mathbf{x}^{\top}\left(\partial_{v} \mathbf{Q}_{v}\right) \mathbf{x}<0, \quad \forall(\mathbf{x}, v) \in \mathcal{H}(\varepsilon) .
$$

Daraus folgt, dass die Bedingung (iii) des Satzes 1 für alle $(\mathbf{x}, v) \in \mathcal{H}(\varepsilon)$ erfüllt ist. Die Bedingung (ii) ist darüber hinaus für alle $\mathbf{x} \in \mathcal{E}_{\mathrm{P}}(1) \backslash \mathcal{E}_{\mathrm{P}}(\varepsilon)$ erfüllt, da

$$
\begin{array}{ll}
\lim _{v \rightarrow 1^{-}} g_{\mathrm{P}}(\mathbf{x}, v)=g_{\mathrm{P}}(\mathbf{x}, 1)<0, & \forall \mathbf{x} \in \mathcal{E}_{\mathrm{P}}(1) \backslash \mathcal{E}_{\mathrm{P}}(\varepsilon), \\
\lim _{v \rightarrow \varepsilon^{+}} g_{\mathrm{P}}(\mathbf{x}, v)=g_{\mathrm{P}}(\mathbf{x}, \varepsilon)>0, & \forall \mathbf{x} \in \mathcal{E}_{\mathrm{P}}(1) \backslash \mathcal{E}_{\mathrm{P}}(\varepsilon) .
\end{array}
$$

Die Bedingungen ( $\mathrm{ii}$ ) und (iii) stellen sicher, dass die Gleichung $g_{\mathrm{P}}(\mathbf{x}, v)=0$ eine eindeutige Lösung für jedes $\mathbf{x} \epsilon$ $\mathcal{E}_{\mathrm{P}}(1) \backslash \mathcal{E}_{\mathrm{P}}(\varepsilon)$ hat, welche eine stetige Funktion $v=v(\mathbf{x})$ für $\varepsilon<v<1$ ist. Darüber hinaus stellt

$$
V_{\mathrm{P}}(\mathbf{x}):= \begin{cases}v(\mathbf{x}), \text { mit } g_{\mathrm{P}}(\mathbf{x}, v)=0, & \mathbf{x} \in \mathcal{E}_{\mathrm{P}}(1) \backslash \mathcal{E}_{\mathrm{P}}(\varepsilon), \\ \varepsilon \mathbf{x}^{\top} \mathbf{Q}_{\varepsilon} \mathbf{x}, & \mathbf{x} \in \mathcal{E}_{\mathrm{P}}(\varepsilon)\end{cases}
$$

eine Ljapunov-Funktion des Systems dar. Dies folgt aus der Anwendung der direkten Methode von Ljapunov. ${ }^{5} \mathrm{Da}-$ bei gilt $V_{\mathrm{P}}(\mathbf{0})=0$, sowie $V_{\mathrm{P}}(\mathbf{x})>0$ für alle $\mathbf{x} \in \mathcal{E}_{\mathrm{P}}(1) \backslash\{\mathbf{0}\}$. Darüber hinaus gilt aufgrund der Bedingungen (iii) und (iv) $\dot{V}_{\mathrm{P}}(\mathbf{x})<0$ für alle $\mathbf{x} \in \mathcal{E}_{\mathrm{P}}(1) \backslash \mathcal{E}_{\mathrm{p}}(\varepsilon)$, sowie aufgrund der Gleichung (16) $\dot{V}_{\mathrm{p}}(\mathbf{x})<0$ für alle $\mathbf{x} \in \mathcal{E}_{\mathrm{p}}(\varepsilon) \backslash\{\mathbf{0}\}$. Folglich ist die Ruhelage $\mathbf{x}_{\mathrm{R}}=\mathbf{0}$ asymptotisch stabil und die Gebiete $\mathcal{E}_{\mathrm{p}}(v)$ für alle $v \in[\varepsilon, 1]$ verschachtelt und kontraktiv invariant.

Die Bedingung aus Gleichung (9) bezüglich der Stellgrößenbeschränkung ist äquivalent zu

$$
|u(\mathbf{x})| \leq \max _{\mathbf{x}^{\top} \mathbf{P}_{v}^{-1} \mathbf{x}=d}\left|\mathbf{k}_{v}^{\top} \mathbf{x}\right| \leq 1, \quad \forall v \in[\varepsilon, 1] .
$$

Der obige maximale Wert ergibt sich durch die Anwendung des Lagrange-Multiplikatorenansatzes zu

$$
\max _{\mathbf{x}^{\top} \mathbf{P}_{v}^{-1} \mathbf{x}=d}\left|\mathbf{k}_{v}^{\top} \mathbf{x}\right|=\max _{\mathbf{x} \in \mathbb{R}^{n}}\left[ \pm \mathbf{k}_{v}^{\top} \mathbf{x}-\lambda\left(\mathbf{x}^{\top} \mathbf{P}_{v}^{-1} \mathbf{x}-d\right)\right]
$$

5 Vgl. z.B. $[3,17]$. 
mit den Optimalitätsbedingungen

$$
\pm \mathbf{k}_{v}-2 \lambda_{\mathrm{opt}} \mathbf{P}_{v}^{-1} \mathbf{x}_{\mathrm{opt}}=\mathbf{0}, \quad \mathbf{x}_{\mathrm{opt}}^{\top} \mathbf{P}_{v}^{-1} \mathbf{x}_{\mathrm{opt}}=d,
$$

d. h. mit

$$
\mathbf{x}_{\mathrm{opt}}= \pm \frac{1}{2 \lambda_{\mathrm{opt}}} \mathbf{P}_{v} \mathbf{k}_{v}, \quad \lambda_{\mathrm{opt}}= \pm \frac{1}{2} \sqrt{\frac{\mathbf{k}_{v}^{\top} \mathbf{P}_{v} \mathbf{k}_{v}}{d}} .
$$

Der maximale Wert ist folglich

$$
\begin{aligned}
& \max _{\mathbf{x} \in \mathbb{R}^{n}}\left[ \pm \mathbf{k}_{v}^{\top} \mathbf{x}-\lambda\left(\mathbf{x}^{\top} \mathbf{P}_{v}^{-1} \mathbf{x}-d\right)\right] \\
& \quad=\max _{\lambda_{\text {opt }}}\left[\frac{\mathbf{k}_{v}^{\top} \mathbf{P}_{v} \mathbf{k}_{v}}{2 \lambda_{\text {opt }}}\right]=\sqrt{d \mathbf{k}_{v}^{\top} \mathbf{P}_{v} \mathbf{k}_{v}}=r \sqrt{d \mathbf{b}^{\top} \mathbf{P}_{v}^{-1} \mathbf{b}}
\end{aligned}
$$

und Gleichung (18) ist äquivalent zu $r \sqrt{d \mathbf{b}^{\top} \mathbf{P}_{v}^{-1} \mathbf{b}} \leq 1, \forall v \in$ $[\varepsilon, 1]$, d.h. zu

$$
\mathbf{b}^{\top} \mathbf{P}_{v}^{-1} \mathbf{b} \leq \frac{1}{d \cdot r^{2}}, \quad \forall v \in[\varepsilon, 1] .
$$

Da $\partial_{v} \mathbf{P}_{v}>\mathbf{0}, \forall v \in[\varepsilon, 1]$, folgt, dass mit steigendem $v$ die Matrixfunktion $\mathbf{P}_{v}$ monoton steigend ist, d.h. für alle $\varepsilon \leq$ $v_{1}<v_{2} \leq 1$ gilt $\mathbf{P}_{v_{1}} \prec \mathbf{P}_{v_{2}}$. Daraus folgt, dass die Matrixfunktion $\mathbf{P}_{v}^{-1}$ monoton fallend ist, ${ }^{6} \mathrm{~d}$. h. für alle $\varepsilon \leq v_{1}<$ $v_{2} \leq 1$ gilt $\mathbf{P}_{v_{1}}^{-1}>\mathbf{P}_{v_{2}}^{-1}$. Daraus folgt, dass die Matrixfunktion $\mathbf{b}^{\top} \mathbf{P}_{v}^{-1} \mathbf{b}$ ebenfalls monoton fallend ist, $\mathbf{d}$. $\mathrm{h}$. für alle $\varepsilon \leq v_{1}<v_{2} \leq 1$ gilt $\mathbf{b}^{\top} \mathbf{P}_{v_{1}}^{-1} \mathbf{b}>\mathbf{b}^{\top} \mathbf{P}_{v_{2}}^{-1} \mathbf{b} .{ }^{7}$ Folglich gilt $\mathbf{b}^{\top} \mathbf{P}_{v}^{-1} \mathbf{b} \leq$ $\mathbf{b}^{\top} \mathbf{P}_{\varepsilon}^{-1} \mathbf{b}, \quad \forall v \in[\varepsilon, 1]$, und daher

$$
\mathbf{b}^{\top} \mathbf{P}_{v}^{-1} \mathbf{b} \leq \mathbf{b}^{\top} \mathbf{P}_{\varepsilon}^{-1} \mathbf{b} \leq \frac{1}{d \cdot r^{2}}, \quad \forall v \in[\varepsilon, 1],
$$

d. h. Gleichung (9) ist erfüllt.

$i) \Rightarrow$ ii) Falls $i)$ gilt, dann ist das Gebiet $\mathcal{E}_{\mathrm{P}}(v)$ für je$\operatorname{des} v \in[\varepsilon, 1]$ kontraktiv invariant für das System $\dot{\mathbf{x}}=(\mathbf{A}-$ $\left.\mathbf{b k}_{v}^{\top}\right) \mathbf{x}$. Die Funktion $W(\mathbf{x}):=\mathbf{x}^{\top} \mathbf{Q}_{v} \mathbf{x}$ ist dabei eine gültige Ljapunov-Funktion des Systems, d. h.

$$
\left(\mathbf{A}-\mathbf{b} \mathbf{k}_{v}^{\top}\right)^{\top} \mathbf{Q}_{v}+\mathbf{Q}_{v}\left(\mathbf{A}-\mathbf{b} \mathbf{k}_{v}^{\top}\right) \prec \mathbf{0}, \quad \forall v \in[\varepsilon, 1]
$$

und das System ist global asymptotisch stabil. Für $\mathbf{P}_{v}=$ $\mathbf{Q}_{v}^{-1}$ folgt

$$
\left(\mathbf{A}-\mathbf{b} \mathbf{k}_{v}^{\top}\right) \mathbf{P}_{v}+\mathbf{P}_{v}\left(\mathbf{A}-\mathbf{b} \mathbf{k}_{v}^{\top}\right)^{\top} \prec \mathbf{0}, \quad \forall v \in[\varepsilon, 1] .
$$

Sei $\mathbf{B}^{\perp} \in \mathbb{R}^{(n-1) \times n}$ eine Basis für den Nullraum von $\mathbf{b}$, sodass $\mathbf{B}^{\perp} \mathbf{b}=\mathbf{0}$ und $\mathbf{B}^{\perp}\left(\mathbf{B}^{\perp}\right)^{\top}>\mathbf{0}$ gilt. Nach Multiplizieren

6 Dies gilt weil $\partial_{v} \mathbf{P}_{v}^{-1}=-\mathbf{P}_{v}^{-1}\left(\partial_{v} \mathbf{P}_{v}\right) \mathbf{P}_{v}^{-1} \prec \mathbf{0}, \forall v \in[\varepsilon, 1]$.

7 Vgl. [4, Proposition 8.6.13, xv)]. der Gleichung (20) mit $\mathbf{B}^{\perp}$ (von links) und $\left(\mathbf{B}^{\perp}\right)^{\top}$ (von rechts), folgt, dass

$$
\begin{aligned}
& \mathbf{B}^{\perp}\left(\mathbf{A}-\mathbf{b} \mathbf{k}_{v}^{\top}\right) \mathbf{P}_{v}\left(\mathbf{B}^{\perp}\right)^{\top}+\mathbf{B}^{\perp} \mathbf{P}_{v}\left(\mathbf{A}-\mathbf{b k}_{v}^{\top}\right)^{\top}\left(\mathbf{B}^{\perp}\right)^{\top} \\
& \quad=\mathbf{B}^{\perp}\left(\mathbf{A P}_{v}+\mathbf{P}_{v} \mathbf{A}^{\top}\right)\left(\mathbf{B}^{\perp}\right)^{\top} \prec \mathbf{0}, \quad \forall v \in[\varepsilon, 1] .
\end{aligned}
$$

Dies ist äquivalent zu dem Sachverhalt, dass für jedes $v \in$ $[\varepsilon, 1]$ ein Skalar $\mu_{v} \in \mathbb{R}$ existiert, ${ }^{8}$ sodass

$$
\mathbf{A P}_{v}+\mathbf{P}_{v} \mathbf{A}^{\top} \prec \mu_{v} \mathbf{b} \mathbf{b}^{\top}
$$

mit

$$
\begin{aligned}
\mu_{v} & >\mathbf{d}^{\top}\left\{\mathbf{L}_{v}-\mathbf{L}_{v}\left(\mathbf{B}^{\perp}\right)^{\top}\left[\mathbf{B}^{\perp} \mathbf{L}_{v}\left(\mathbf{B}^{\perp}\right)^{\top}\right]^{-1} \mathbf{B}^{\perp} \mathbf{L}_{v}\right\} \mathbf{d}, \\
\mathbf{L}_{v} & =\mathbf{A P}_{v}+\mathbf{P}_{v} \mathbf{A}^{\top}, \\
\mathbf{d}^{\top} & :=\left|b_{r}\right|^{-1} \mathbf{b}_{l}^{+}, \quad b_{r} \in \mathbb{R} \backslash\{\mathbf{0}\}, \mathbf{b}_{l}^{+} \in \mathbb{R}^{1 \times n},
\end{aligned}
$$

wobei das Tupel $\left(\mathbf{b}_{l}, b_{r}\right)$ eine Voll-Rang-Faktorisierung des Vektors b ist. Gleichung (12) folgt durch Skalierung der Matrix $\mathbf{P}_{v}$ durch einen Skalar

$$
\mu>\max \left\{0, \max _{v \in[\varepsilon, 1]} \mu_{v}\right\} \text {. }
$$

Darüber hinaus sind die Gebiete $\mathcal{E}_{\mathrm{P}}(v)$ verschachtelt, d. h.

$$
\begin{aligned}
\mathcal{E}_{\mathrm{P}}\left(v_{2}\right) & \subset \mathcal{E}_{\mathrm{P}}\left(v_{1}\right), \quad \forall \varepsilon \leq v_{2}<v_{1} \leq 1, \\
\partial \mathcal{E}_{\mathrm{P}}\left(v_{2}\right) \cap \partial \mathcal{E}_{\mathrm{P}}\left(v_{1}\right) & =\emptyset, \quad \forall \varepsilon \leq v_{2}<v_{1} \leq 1 .
\end{aligned}
$$

Dies bedeutet, dass für $\mathbf{x} \in \partial \mathcal{E}_{\mathrm{P}}\left(v_{1}\right)$ folgt $g_{\mathrm{P}}\left(\mathbf{x}, v_{1}\right)=0$ und $g_{\mathrm{P}}\left(\mathbf{x}, v_{2}\right)>0, \forall \varepsilon \leq v_{2}<v_{1} \leq 1$, und daher, dass

$$
g_{\mathrm{P}}\left(\mathbf{x}, v_{2}\right)>g_{\mathrm{P}}\left(\mathbf{x}, v_{1}\right), \forall \varepsilon \leq v_{2}<v_{1} \leq 1, \mathbf{x} \in \partial \mathcal{E}_{\mathrm{P}}\left(v_{1}\right)
$$

Folglich gilt

$$
\partial_{v} g_{\mathrm{P}}(\mathbf{x}, v)=\mathbf{x}^{\top}\left(\partial_{v} \mathbf{Q}_{v}\right) \mathbf{x}<0, \forall \mathbf{x} \in \partial \mathcal{E}_{\mathrm{P}}(v), v \in[\varepsilon, 1]
$$

und, somit, dass $\partial_{v} \mathbf{Q}_{v} \prec \mathbf{0}, \forall v \in[\varepsilon, 1]$. Daraus folgt, dass

$$
\partial_{v} \mathbf{P}_{v}=\partial_{v}\left(\mathbf{Q}_{v}^{-1}\right)=-\mathbf{Q}_{v}^{-1}\left(\partial_{v} \mathbf{Q}_{v}\right) \mathbf{Q}_{v}^{-1}>\mathbf{0}, \forall v \in[\varepsilon, 1],
$$

d.h. es folgt Gleichung (11). Gleichung (10) und die polynomiale Form der Matrix $\mathbf{P}_{v}$ sind durch $\mathbf{P}_{v}=\mathbf{Q}_{v}^{-1}=\mathbf{R}_{v} \succ$ 0 sichergestellt. Die Bedingungen (10)-(12) sind somit auch notwendig für die Existenz einer WSVR mit inverspolynomialen Selektionsstrategien.

Satz 3 stellt also die hinreichenden und notwendigen Bedingungen für die Existenz einer stabilisierenden WSVR

8 Vgl. Finsler's Lemma, [16, Theorem 2.3.10]. 
mit invers-polynomialen Selektionsstrategien dar. Die Stabilisierbarkeitsbedingungen sind somit nicht konservativ. Die damit entworfenen Regelgesetze können als Startwerte für eine Regleroptimierung verwendet werden. Dies wird im Folgenden im Falle der Maximierung der Konvergenzrate gezeigt.

\section{Konvergenzoptimale Bang-Bang-WSVR}

Wie in [9, S. 230] gezeigt, kann man eine untere Grenze der Konvergenzrate eines nichtlinearen Systems anhand der Abklingrate einer für das System gültigen LjapunovFunktion untersuchen. In diesem Abschnitt wird das Regelgesetz bestimmt, das eine solche Abklingrate maximiert. Es wird gezeigt, dass das resultierende Regelgesetz ein Bang-Bang-Regelgesetz mit einer parameterabhängigen Umschaltstrategie ist, wobei diese abhängig von der gewählten Ljapunov-Funktion ist.

Unter der Voraussetzung, dass die Selektionsstrategie $\mathcal{g}_{\mathrm{u}}(\mathbf{x}, v)=0$ aus Gleichung (4) eine eindeutige und stetige Lösung $v(\mathbf{x}): \mathcal{U}_{0} \backslash\{\mathbf{0}\} \rightarrow(\varepsilon, 1)$ für jedes $\mathbf{x} \in \mathcal{U}_{0} \backslash\{\mathbf{0}\}$ aus einer Umgebung der Ruhelage hat, kann diese Umgebung in die ellipsoidalen Gebiete

$$
\mathcal{E}_{\mathrm{u}}(v):=\left\{\mathbf{x} \in \mathbb{R}^{n} \mid \mathbf{x}^{\top} \mathbf{Q}_{v} \mathbf{x}<1\right\}
$$

geteilt werden, wobei jedem Gebiet ein eindeutiges $v \in$ $(\varepsilon, 1)$ zugewiesen wird. Somit kann für die Optimierung der Konvergenzrate des Gesamtsystems die Funktion

$$
V_{\mathrm{u}}(\mathbf{x}):= \begin{cases}v(\mathbf{x}), \text { mit } g_{u}(\mathbf{x}, v)=0, & \mathbf{x} \in \mathcal{E}_{\mathrm{u}}(1) \backslash \mathcal{E}_{u}(\varepsilon) \\ \varepsilon \mathbf{x}^{\top} \mathbf{Q}_{\varepsilon} \mathbf{x}, & \mathbf{x} \in \mathcal{E}_{\mathrm{u}}(\varepsilon)\end{cases}
$$

verwendet werden. Diese Funktion ist für alle $\mathbf{x} \in$ $\mathcal{E}_{\mathrm{u}}(1) \backslash\{\boldsymbol{0}\}$ positiv definit. Entlang einer Trajektorie des Systems aus Gleichung (2) gilt

$$
\dot{V}_{\mathrm{u}}(\mathbf{x}, u)= \begin{cases}V_{\mathrm{u}, 1}(\mathbf{x}, u), & \mathbf{x} \in \mathcal{E}_{\mathrm{u}}(1) \backslash \mathcal{E}_{\mathrm{u}}(\varepsilon) \\ V_{\mathrm{u}, 2}(\mathbf{x}, u), & \mathbf{x} \in \mathcal{E}_{\mathrm{u}}(\varepsilon) \backslash\{\mathbf{0}\}\end{cases}
$$

mit $^{9}$

$$
\begin{aligned}
& V_{\mathrm{u}, 1}(\mathbf{x}, u)=-\frac{\mathbf{x}^{\top}\left(\mathbf{A}^{\top} \mathbf{Q}_{v}+\mathbf{Q}_{v} \mathbf{A}\right) \mathbf{x}+2 \mathbf{x}^{\top} \mathbf{Q}_{v} \mathbf{b} u}{\mathbf{x}^{\top}\left(\partial v \mathbf{Q}_{v}\right) \mathbf{x}}, \\
& V_{\mathrm{u}, 2}(\mathbf{x}, u)=\varepsilon \mathbf{x}^{\top}\left(\mathbf{A}^{\top} \mathbf{Q}_{\varepsilon}+\mathbf{Q}_{\varepsilon} \mathbf{A}\right) \mathbf{x}+2 \mathbf{x}^{\top} \mathbf{Q}_{\varepsilon} \mathbf{b} u .
\end{aligned}
$$

9 Die Funktion $V_{\mathrm{u}, 1}(\mathbf{x}, u)$ ergibt sich dabei aus der Zeitableitung der impliziten Funktion $v(\mathbf{x})$ aus Gleichung (1).
Daraus folgt, dass für jedes $\mathbf{x} \in \mathcal{E}_{\mathrm{u}}(1) \backslash\{\mathbf{0}\}$

$$
\arg \min _{|u| \leq 1} \dot{V}_{\mathrm{u}}(\mathbf{x}, u)=-\operatorname{sgn}\left(\mathbf{b}^{\top} \mathbf{Q}_{v} \mathbf{x}\right)
$$

gilt, d.h., das Regelgesetz, das die Funktion $\dot{V}_{\mathrm{u}}(\mathbf{x}, u)$ in jedem Punkt $\mathbf{x} \in \mathcal{E}_{\mathrm{u}}(\mathbf{1}) \backslash\{\mathbf{0}\}$ minimiert, ein Bang-Bangartiges Regelgesetz mit einer parameterabhängigen Umschaltstrategie ist. Die Stabilitätsbedingungen dieses Regelgesetzes werden in Abschnitt 3.1 analysiert. Dabei wird ersichtlich, dass die Existenz eines stabilisierenden beschränkten Reglers, wie z.B. in Abschnitt 2 bestimmt, notwendig und hinreichend für die Stabilität der konvergenzoptimalen Regelung ist. Der Entwurf der konvergenzoptimalen Regelung ist also auch nicht konservativ. Jedoch hat die Unstetigkeit des Regelgesetzes Nachteile in einer praktischen Implementierung, wie z.B. die ununterbrochene Aktivität des Reglers aufgrund unvermeidbaren Rauschens. Daher wird eine stetige Approximation des konvergenzoptimalen Regelgesetzes in Abschnitt 3.2 untersucht, welche auf Kosten einer geringeren Konvergenzrate einen stetigen Verlauf erzielt.

\subsection{Existenzbedingungen des konvergenzoptimalen Regelgesetzes}

Das folgende Theorem stellt die notwendigen und hinreichenden Stabilitätsbedingungen für das konvergenzoptimale Regelgesetz aus Gleichung (21) vor. Dieses Theorem baut auf das Theorem 1 aus [8] auf. Letzteres untersucht die Stabilitätsbedingungen eines konvergenzoptimalen Reglers mit einer parameterunabhängigen, zustandslinearen Umschaltstrategie ${ }^{10}$, wohingegen das im Folgenden vorgestellte Theorem die Stabilitätsbedingungen eines konvergenzoptimalen Reglers mit einer parameterabhängigen Umschaltstrategie untersucht. Darüber hinaus stellt dieses Theorem eine Generalisierung des Theorems aus [15] dar, welches die Stabilitätsbedingungen eines konvergenzoptimalen Reglers mit der klassischen WSVR ${ }^{11}$ aus [2] untersucht. Das im Folgenden vorgestellte Theorem gilt für quadratische Selektionsgleichungen der Form $\mathbf{x}^{\top} \mathbf{Q}_{v} \mathbf{x}-1=$ 0 mit beliebigen parameterabhängigen Matrizen $\mathbf{Q}_{v}>\mathbf{0}$.

Satz 5. Gegeben sei das LTI-System aus Gleichung (2) und eine reelle Zahl $\varepsilon \in(0,1)$. Im Folgenden verwenden wir die

10 Das konvergenzoptimale Regelgesetz aus [8] lautet $u=$ $-\operatorname{sgn}\left(\mathbf{b}^{\top} \mathbf{Q} \mathbf{x}\right)$, mit einer konstanten Matrix $\mathbf{Q}>\mathbf{0}$.

11 Die Selektionsgleichung der klassischen WSVR lautet $\mathbf{x}^{\top} \mathbf{Q}_{v} \mathbf{x}=1$, mit der Matrix $\mathbf{Q}_{v}=\mathbf{D}_{v}^{-1} \mathbf{Q}_{1} \mathbf{D}_{v}^{-1}>\mathbf{0}$ und $\mathbf{D}_{v}=\operatorname{diag}\left(v^{n},, v\right)$. 
Notation

$$
\mathcal{H}(\varepsilon):=\left\{(\mathbf{x}, v) \mid \mathbf{x} \in \mathcal{U}_{0} \backslash\{\mathbf{0}\}, \varepsilon<v<1\right\},
$$

wobei $\mathcal{U}_{0} \subseteq \mathcal{B}_{\delta}(\mathbf{0})$ eine noch zu bestimmende Umgebung der Ruhelage $\mathbf{x}_{\mathrm{R}}=\mathbf{0}$ ist. Die folgenden Aussagen sind äquivalent:

a) Es existiert ein beschränktes Regelgesetz

$$
u_{f}=-f(\mathbf{x}, v), \quad\left|u_{f}\right| \leq 1,
$$

eine Umgebung $\mathcal{U}_{0} \subseteq \mathcal{B}_{\delta}(\mathbf{0})$ der Ruhelage, sowie eine Matrix $\mathbf{Q}_{v}>\mathbf{0}, \forall v \in(\varepsilon, 1)$, sodass die Funktion

$$
g_{f}(\mathbf{x}, v): \mathcal{H}(\varepsilon) \rightarrow \mathbb{R}, \quad g_{f}(\mathbf{x}, v):=\mathbf{x}^{\top} \mathbf{Q}_{v} \mathbf{x}-1,
$$

für alle $(\mathbf{x}, v) \in \mathcal{H}(\varepsilon)$ die Bedingungen (ii)-(iv) des Satzes 1 für das System (2) mit dem Regelgesetz (22) erfüllt.

b) Es existiert eine Umgebung der Ruhelage $\mathcal{U}_{0} \subseteq \mathcal{B}_{\delta}(\mathbf{0})$ und eine Matrix $\mathbf{Q}_{v}>\mathbf{0}, \forall v \in(\varepsilon, 1)$, sodass die Funktion

$$
g_{s}(\mathbf{x}, v): \mathcal{H}(\varepsilon) \rightarrow \mathbb{R}, \quad g_{s}(\mathbf{x}, v):=\mathbf{x}^{\top} \mathbf{Q}_{v} \mathbf{x}-1,
$$

für alle $(\mathbf{x}, v) \in \mathcal{H}(\varepsilon)$ die Bedingungen (ii)-(iv) des Satzes 1 für das System (2) mit dem Regelgesetz

$$
u_{s}=-\operatorname{sgn}\left(\mathbf{b}^{\top} \mathbf{Q}_{v} \mathbf{x}\right) \text {. }
$$

erfüllt.

c) Es existiert eine Funktion $\kappa_{v}:[\varepsilon, 1] \rightarrow \mathbb{R}_{+}$, eine Umgebung der Ruhelage $\mathcal{U}_{0} \subseteq \mathcal{B}_{\delta}(\mathbf{0})$, sowie eine Matrix $\mathbf{Q}_{v}>$ $\mathbf{0}, \forall v \in(\varepsilon, 1)$, sodass die Funktion

$$
g_{\text {sat }}(\mathbf{x}, v): \mathcal{H}(\varepsilon) \rightarrow \mathbb{R}, \quad g_{\text {sat }}(\mathbf{x}, v):=\mathbf{x}^{\top} \mathbf{Q}_{v} \mathbf{x}-1,
$$

für alle $(\mathbf{x}, v) \in \mathcal{H}(\varepsilon)$ die Bedingungen (ii)-(iv) des Satzes 1 für das System (2) mit dem Regelgesetz

$$
u_{\text {sat }}=-\operatorname{sat}\left(\kappa_{v} \mathbf{b}^{\top} \mathbf{Q}_{v} \mathbf{x}\right),
$$

erfüllt.

Beweis. $a) \Rightarrow b$ ) Falls $a$ ) gilt, dann ist die Ableitung der Funktion $g_{s}(\mathbf{x}, v)$ entlang der Trajektorien des Systems (2) mit dem Regelgesetz (25)

$$
\begin{aligned}
& \partial_{t} g_{s}(\mathbf{x}(t), v)=\mathbf{x}^{\top}\left(\mathbf{A}^{\top} \mathbf{Q}_{v}+\mathbf{Q}_{v} \mathbf{A}\right) \mathbf{x}-2\left|\mathbf{x}^{\top} \mathbf{Q}_{v} \mathbf{b}\right| \\
& =\partial_{t} g_{f}(\mathbf{x}(t), v)+2 \mathbf{x}^{\top} \mathbf{Q}_{v} \mathbf{b} f(\mathbf{x}, v)-2\left|\mathbf{x}^{\top} \mathbf{Q}_{v} \mathbf{b}\right| \\
& =\partial_{t} g_{f}(\mathbf{x}(t), v)+2\left|\mathbf{x}^{\top} \mathbf{Q}_{v} \mathbf{b}\right| \cdot\left[\operatorname{sgn}\left(\mathbf{x}^{\top} \mathbf{Q}_{v} \mathbf{b}\right) f(\mathbf{x}, v)-1\right] \\
& =\partial_{t} g_{f}(\mathbf{x}(t), v) \\
& +2\left|\mathbf{b}^{\top} \mathbf{Q}_{v} \mathbf{x}\right| \cdot\left\{\begin{array}{l}
-|f(\mathbf{x}, v)|-1, \operatorname{sgn}(f(\mathbf{x}, v)) \neq \operatorname{sgn}\left(\mathbf{x}^{\top} \mathbf{Q}_{v} \mathbf{b}\right) \\
|f(\mathbf{x}, v)|-1, \operatorname{sonst}
\end{array}\right. \\
& <0, \forall(\mathbf{x}, v) \in \mathcal{H}(\varepsilon),
\end{aligned}
$$

wobei $\partial_{t} g_{f}(\mathbf{x}(t), v)$ die Ableitung der Funktion $g_{f}(\mathbf{x}, v)$ entlang der Trajektorien des Systems (2) mit dem Regelgesetz (22) darstellt und negativ für alle $(\mathbf{x}, v) \in \mathcal{H}(\varepsilon)$ ist. Das bedeutet, dass die Bedingung (iv) erfüllt ist. Darüber hinaus folgt unmittelbar aus $a$ ), dass die Bedingungen (ii)(iii) für alle $(\mathbf{x}, v) \in \mathcal{H}(\varepsilon)$ für das System (2) mit dem Regelgesetz (25) erfüllt sind.

$b) \Rightarrow c$ ) Für diesen Teil des Beweises verwenden wir Finsler's Lemma ${ }^{12}$. Sei

$$
\mathbf{s}_{v}:=\mathbf{Q}_{v} \mathbf{b}, \quad \mathbf{s}_{v}:(\varepsilon, 1) \rightarrow \mathbb{R}^{n},
$$

mit $\operatorname{Rang}\left(\mathbf{s}_{v}\right)=1$, und $\mathbf{S}_{v}^{\perp} \in \mathbb{R}^{(n-1) \times n}$ eine Basis des Nullraumes von $\mathbf{s}_{v}^{\top}$, sodass $\mathbf{S}_{v}^{\perp} \mathbf{s}_{v}=\mathbf{0}$ und $\mathbf{S}_{v}^{\perp}\left(\mathbf{S}_{v}^{\perp}\right)^{\top}>\mathbf{0} .{ }^{13}$ Wenn b) gilt, dann folgt aus der Bedingung (iv) des Satzes 1, dass

$$
\partial_{t} g_{s}(\mathbf{x}(t), v)=\mathbf{x}^{\top}\left(\mathbf{A}^{\top} \mathbf{Q}_{v}+\mathbf{Q}_{v} \mathbf{A}\right) \mathbf{x}-2\left|\mathbf{x}^{\top} \mathbf{Q}_{v} \mathbf{b}\right|<0,
$$

$\forall(\mathbf{x}, v) \in \mathcal{H}(\varepsilon)$. Für jedes $\mathbf{x} \in \mathcal{N}\left(\mathbf{s}_{v}^{\top}\right):=\left\{\mathbf{x} \in \mathbb{R}_{*}^{n} \mid \mathbf{s}_{v}^{\top} \mathbf{x}=0\right\}$ gilt

$$
\mathbf{x}^{\top}\left(\mathbf{A}^{\top} \mathbf{Q}_{v}+\mathbf{Q}_{v} \mathbf{A}\right) \mathbf{x}<0
$$

$\forall(\mathbf{x}, v) \in\left\{(\mathbf{x}, v) \mid \mathbf{x} \in \mathcal{N}\left(\mathbf{s}_{v}^{\top}\right), v \in(\varepsilon, 1)\right\}$. Dabei existiert für jedes $\mathbf{x} \in \mathcal{N}\left(\mathbf{s}_{v}^{\top}\right)$ ein $\mathbf{y} \in \mathbb{R}_{*}^{n-1}$, sodass

$$
\mathbf{x}=\left(\mathbf{S}_{v}^{\perp}\right)^{\top} \mathbf{y}
$$

Daraus folgt, dass

$$
\begin{aligned}
\mathbf{y}^{\top} \mathbf{S}_{v}^{\perp} \mathbf{L}_{v}\left(\mathbf{S}_{v}^{\perp}\right)^{\top} \mathbf{y}<0, \\
\forall(\mathbf{y}, v) \in\left\{(\mathbf{y}, v) \mid \mathbf{y} \in \mathbb{R}_{*}^{n-1}, v \in(\varepsilon, 1)\right\},
\end{aligned}
$$

mit $\mathbf{L}_{v}:=\mathbf{A}^{\top} \mathbf{Q}_{v}+\mathbf{Q}_{v} \mathbf{A}$. Daraus folgt, dass

$$
\mathbf{S}_{v}^{\perp} \mathbf{L}_{v}\left(\mathbf{S}_{v}^{\perp}\right)^{\top} \prec 0, \quad \forall v \in(\varepsilon, 1) .
$$

Im Folgenden wenden wir eine Kongruenztransformation der Matrix $2 \kappa_{v} \mathbf{s}_{v} \mathbf{s}_{v}^{\top}-\mathbf{L}_{v}$ an, wobei $\mu_{v}:=2 \kappa_{v}$. Sei $\mathbf{T}_{v} \in \mathbb{R}^{n \times n}$ eine nichtsinguläre Matrix, definiert als

$$
\mathbf{T}_{v}:=\left[\begin{array}{ll}
\mathbf{d}_{v} & \left(\mathbf{S}_{v}^{\perp}\right)^{\top}
\end{array}\right]^{\top}
$$

mit dem Vektor $\mathbf{d}_{v} \in \mathbb{R}^{n}$ definiert als $\mathbf{d}_{v}^{\top}:=s_{v_{r}}^{-1} \mathbf{s}_{v_{l}}^{+}$, wobei das Tupel $\left(s_{v_{l}}, s_{v_{r}}\right)$ eine Voll-Rang-Faktorisierung ${ }^{14}$ des Vektors

12 Vgl. [16, Theorem 2.3.10].

13 Eine notwendige und hinreichende Bedingung, dass $\mathbf{S}_{v}^{\perp}\left(\mathbf{S}_{v}^{\perp}\right)^{\top}>\mathbf{0}$ gilt, ist, dass $\operatorname{Rang}\left(\mathbf{S}_{v}^{\perp}\right)=n-1$ ist. Dies resultiert aus der Tatsache, dass $\mathbf{S}_{v}^{\perp}\left(\mathbf{S}_{v}^{\perp}\right)^{\top}>\mathbf{0} \Leftrightarrow \lambda\left(\mathbf{S}_{v}^{\perp}\left(\mathbf{S}_{v}^{\perp}\right)^{\top}\right)>0 \Leftrightarrow \operatorname{Rang}\left(\mathbf{S}_{v}^{\perp}\left(\mathbf{S}_{v}^{\perp}\right)^{\top}\right)=\operatorname{Rang}\left(\mathbf{S}_{v}^{\perp}\right)=n-1$.

14 Vgl. Def. 3. 
$\mathbf{s}_{v}$ ist. ${ }^{15}$ Daraus folgt, dass

$$
\begin{aligned}
& \mathbf{T}_{v}\left(\mu_{v} \mathbf{s}_{v} \mathbf{s}_{v}^{\top}-\mathbf{L}_{v}\right) \mathbf{T}_{v}^{\top} \\
& =\left[\begin{array}{c}
\mathbf{d}_{v}^{\top} \\
\mathbf{S}_{v}^{\perp}
\end{array}\right]\left(\mu_{v} \mathbf{s}_{v} \mathbf{s}_{v}^{\top}-\mathbf{L}_{v}\right)\left[\begin{array}{ll}
\mathbf{d}_{v} & \left(\mathbf{S}_{v}^{\perp}\right)^{\top}
\end{array}\right] \\
& =\left[\begin{array}{cc}
\mathbf{d}_{v}^{\top}\left(\mu_{v} \mathbf{s}_{v} \mathbf{s}_{v}^{\top}-\mathbf{L}_{v}\right) \mathbf{d}_{v} & \mathbf{d}_{v}^{\top}\left(\mu_{v} \mathbf{s}_{v} \mathbf{s}_{v}^{\top}-\mathbf{L}_{v}\right)\left(\mathbf{S}_{v}^{\perp}\right)^{\top} \\
\mathbf{S}_{v}^{\perp}\left(\mu_{v} \mathbf{s}_{v} \mathbf{s}_{v}^{\top}-\mathbf{L}_{v}\right) \mathbf{d}_{v} & \mathbf{S}_{v}^{\perp}\left(\mu_{v} \mathbf{s}_{v} \mathbf{s}_{v}^{\top}-\mathbf{L}_{v}\right)\left(\mathbf{S}_{v}^{\perp}\right)^{\top}
\end{array}\right] \\
& =\left[\begin{array}{cc}
\mu_{v}-\mathbf{d}_{v}^{\top} \mathbf{L}_{v} \mathbf{d}_{v} & -\mathbf{d}_{v}^{\top} \mathbf{L}_{v}\left(\mathbf{S}_{v}^{\perp}\right)^{\top} \\
-\mathbf{S}_{v}^{\perp} \mathbf{L}_{v} \mathbf{d}_{v} & -\mathbf{S}_{v}^{\perp} \mathbf{L}_{v}\left(\mathbf{S}_{v}^{\perp}\right)^{\top}
\end{array}\right] .
\end{aligned}
$$

Da $-\mathbf{S}_{v}^{\perp} \mathbf{L}_{v}\left(\mathbf{S}_{v}^{\perp}\right)^{\top}>\mathbf{0}$, ist die Blockmatrix aus Gleichung (29) positiv definit dann und nur dann, wenn für jedes $v \in(\varepsilon, 1)$ ein Skalar $\mu_{v} \in \mathbb{R}$ existiert, sodass $^{16}$

$$
\mu_{v}>\mathbf{d}_{v}^{\top}\left[\mathbf{L}_{v}-\mathbf{L}_{v}\left(\mathbf{S}_{v}^{\perp}\right)^{\top}\left(\mathbf{S}_{v}^{\perp} \mathbf{L}_{v}\left(\mathbf{S}_{v}^{\perp}\right)^{\top}\right)^{-1} \mathbf{S}_{v}^{\perp} \mathbf{L}_{v}\right] \mathbf{d}_{v} .
$$

In diesem Fall ist die Matrix $\mu_{v} \mathbf{s}_{v} \mathbf{s}_{v}^{\top}-\mathbf{L}_{v}$ auch positiv definit, da beide Matrizen kongruent sind. Für $\kappa_{v}=0.5 \mu_{v}$ folgt, dass

$$
\mathbf{A}^{\top} \mathbf{Q}_{v}+\mathbf{Q}_{v} \mathbf{A}-2 \kappa_{v} \mathbf{Q}_{v} \mathbf{b b}^{\top} \mathbf{Q}_{v} \prec \mathbf{0} .
$$

Daraus folgt, dass die Funktion $g_{\text {sat }}(\mathbf{x}, v)$ die Bedingung (iv) für das System (2) mit dem Regelgesetz $u_{\kappa}:=-\kappa_{v} \mathbf{b}^{\top} \mathbf{Q}_{v} \mathbf{x}$ erfüllt. Da diese Bedingung auch mit dem beschränkten Regelgesetz $u_{s}$ erfüllt ist, folgt $c$ ) aus der Anwendung des Theorems 2 aus [14]. Darüber hinaus folgt unmittelbar aus $b)$, dass die Bedingungen (ii)-(iii) für alle $(\mathbf{x}, v) \in \mathcal{H}(\varepsilon)$ für das System (2) mit dem Regelgesetz (27) erfüllt sind.

c) $\Rightarrow a$ ) ist offensichtlich.

Korollar 6. Für das System aus Gleichung (2) und das Regelgesetz aus Gleichung (22), (25) oder (27) seien die Bedingungen $(i i)-(i v)$ des Satzes 1 für alle $(\mathbf{x}, v) \in \mathcal{H}(\varepsilon)$ mit $\mathcal{U}_{0}=\mathcal{E}_{\mathrm{u}}(1) \backslash \mathcal{E}_{\mathrm{u}}(\varepsilon)$ erfüllt, wobei

$$
\mathcal{E}_{\mathrm{u}}(v):=\left\{\mathbf{x} \in \mathbb{R}^{n} \mid \mathbf{x}^{\top} \mathbf{Q}_{v} \mathbf{x}-1<0\right\} .
$$

Auf Grund des Satzes 5 ist dies z.B. der Fall wenn die Bedingungen (10)-(12) aus Satz 3 erfüllt sind. Dann ist das Gebiet $\mathcal{E}_{\mathrm{u}}(1)$ kontraktiv invariant. Darüber hinaus konvergieren die Trajektorien, die in dem Gebiet starten, asymptotisch gegen die Ruhelage.

15 Die Matrix $\mathbf{T}_{v}$ ist nichtsingulär, da $\mathcal{N}\left(\mathbf{d}_{v}\right) \cap \mathcal{N}\left(\mathbf{S}_{v}^{\perp}\right)=\{0\}$ gilt, vgl. dazu [4, Fakt 2.11.3]. Dies ist ersichtlich aus der Tatsache, dass per Definition $\mathbf{S}_{v}^{\perp} \mathbf{s}_{v}=\mathbf{0}$ gilt, und daher $\mathbf{s}_{v} \in \mathcal{N}\left(\mathbf{S}_{v}^{\perp}\right)$. Da noch $\mathbf{d}_{v}^{\top} \mathbf{s}_{v}=\mathbf{s}_{v}^{\top} \mathbf{s}_{v} /\left\|\mathbf{s}_{v}\right\|^{2}=$ $1 \neq 0, \forall v \in[\varepsilon, 1]$, folgt, dass $\mathbf{s}_{v} \notin \mathcal{N}\left(\mathbf{s}_{v}^{\top}\right)$.

16 Dies resultiert aus der Bedingung, dass das Schur-Komplement von $-\mathbf{S}_{v}^{\perp} \mathbf{L}_{v}\left(\mathbf{S}_{v}^{\perp}\right)^{\top}$ bezüglich der Blockmatrix aus Gleichung (29) positiv definit sein muss, vgl. [4, Proposition 8.2.4].
Beweis. Sei die Funktion

$$
V_{\mathrm{o}}(\mathbf{x}):= \begin{cases}v(\mathbf{x}), \text { mit } g_{\mathrm{o}}(\mathbf{x}, v)=0, & \mathbf{x} \in \mathcal{E}_{\mathrm{u}}(1) \backslash \mathcal{E}_{\mathrm{u}}(\varepsilon), \\ \varepsilon \mathbf{x}^{\top} \mathbf{Q}_{\varepsilon} \mathbf{x}, & \mathbf{x} \in \mathcal{E}_{\mathrm{u}}(\varepsilon),\end{cases}
$$

wobei die Bezeichnung $(\cdot)_{\mathrm{o}}$ für eins der Symbole $(\cdot)_{\mathrm{f}},(\cdot)_{\mathrm{s}}$ oder $(\cdot)_{\text {sat }}$ steht. Dabei gilt

$$
\begin{array}{ll}
V_{\mathrm{o}}(\mathbf{0})=0, & \\
V_{\mathrm{o}}(\mathbf{x})>0, & \forall \mathbf{x} \in \mathcal{E}_{\mathrm{u}}(1) \backslash\{\mathbf{0}\}, \\
\dot{V}_{\mathrm{o}}(\mathbf{x})<0, & \forall \mathbf{x} \in \mathcal{E}_{\mathrm{u}}(1) \backslash\{\mathbf{0}\} .
\end{array}
$$

Gleichung (32) folgt aus der Definition der Funktion $V_{\mathrm{o}}(\mathbf{x})$ aus Gleichung (31). Gleichung (33) folgt aus $v \in(\varepsilon, 1)$ und $\mathbf{Q}_{\varepsilon}>\mathbf{0}$. Für alle $\mathbf{x} \in \mathcal{E}_{\mathrm{u}}(1) \backslash\{\mathbf{0}\}$ folgt Gleichung (34) aus der kontraktiven Invarianz der Gebiete $\mathcal{E}_{\mathrm{u}}(v)$ für alle $v \in[\varepsilon, 1]$. Letzteres folgt aus Satz 1 und Korollar 2. Somit ist das Ellipsoid $\mathcal{E}_{\mathrm{u}}(1)$ kontraktiv invariant. ${ }^{17}$

Schließlich wird die Berechnung der notwendigen Verstärkung $\kappa_{v}$, bzw. der unteren Grenze der Funktion $\mu_{v}$ aus Gleichung (30), gezeigt.

\subsection{Berechnung des Parameters $\kappa_{v}$}

Um die untere Grenze der Funktion $\mu_{v}$ aus Gleichung (30) $\mathrm{zu}$ berechnen, muss eine Basis des Nullraumes der Umschaltfunktion $\mathbf{s}_{v}^{\top}=\mathbf{b}^{\top} \mathbf{Q}_{v}$, mit $\operatorname{Rang}\left(\mathbf{s}_{v}\right)=1, \forall v \in[\varepsilon, 1]$, berechnet werden. Die in diesem Beitrag analysierte Form der parameterabhängigen Matrix $\mathbf{Q}_{v}$ ist gegeben durch

$$
\mathbf{Q}_{v}:=\mathbf{R}_{v}^{-1}=\frac{1}{\operatorname{det}\left(\mathbf{R}_{v}\right)} \mathbf{R}_{v}^{A},
$$

mit

$$
\mathbf{R}_{v}:=\sum_{i=\mathrm{M}_{\mathrm{L}}}^{\mathrm{M}_{\mathrm{U}}} v^{i} \mathbf{R}_{c_{i}}=v^{\mathrm{M}_{\mathrm{L}}} \sum_{i=0}^{l} v^{i} \mathbf{R}_{c_{i+\mathrm{M}_{\mathrm{L}}}}>\mathbf{0}, \forall v \in[\varepsilon, 1],
$$

wobei $\mathbf{R}_{c_{i}} \in$ Sym $^{n}$ und $l:=\mathrm{M}_{\mathrm{U}}-\mathrm{M}_{\mathrm{L}}$.

In [11, Lemma A.1] wurde eine obere Grenze des Grades der Adjunkten einer polynomialen Matrix nachgewiesen. Für die Adjunkte der polynomialen Matrix aus Gleichung (35) gilt demnach

$$
\begin{aligned}
\mathbf{R}_{v}^{A} & =v^{\mathrm{M}_{\mathrm{L}} \cdot(n-1)}\left(\sum_{i=0}^{l} v^{i} \mathbf{R}_{c_{i+\mathrm{M}_{\mathrm{L}}}}\right)^{A} \\
& =v^{\mathrm{M}_{\mathrm{L}} \cdot(n-1)} \sum_{i=0}^{\mu} v^{i} \mathbf{N}_{c_{i}}, \quad \mathbf{N}_{c_{i}} \in \mathrm{Sym}^{n},
\end{aligned}
$$

17 Vgl. Def. 1. 
wobei

$$
\mu \leq l \cdot \min \{n-1, n-q\}, \quad q:=\operatorname{dim}\left[\bigcap_{i=1}^{l} \mathcal{N}\left(\mathbf{R}_{\mathrm{c}_{i+\mathrm{M}_{\mathrm{L}}}}\right)\right] .
$$

Folglich gilt

$$
\mathbf{s}_{v}=\frac{v^{\mathrm{M}_{\mathrm{L}} \cdot(n-1)}}{\operatorname{det}\left(\mathbf{R}_{v}\right)} \sum_{i=0}^{\mu} v^{i} \mathbf{s}_{c_{i}},
$$

mit $\mathbf{s}_{c_{i}}:=\mathbf{N}_{c_{i}}$ b. Gesucht wird eine polynomiale Matrix $\mathbf{S}_{v}^{\perp} \in$ $\mathbb{R}^{(n-1) \times n}$, wofür

$$
\begin{aligned}
\mathbf{S}_{v}^{\perp} \mathbf{S}_{v} & =\mathbf{0}, \quad \forall v \in[\varepsilon, 1], \\
\mathbf{S}_{v}^{\perp}\left(\mathbf{S}_{v}^{\perp}\right)^{\top}>\mathbf{0}, & \forall v \in[\varepsilon, 1],
\end{aligned}
$$

gilt. Diese wird in der Form

$$
\mathbf{S}_{v}^{\perp}=\sum_{i=0}^{v} v^{i} \mathbf{S}_{c_{i}}^{\perp}, \quad \mathbf{S}_{c_{i}}^{\perp} \in \mathbb{R}^{(n-1) \times n}, v>0,
$$

angenommen. Sind die Koeffizienten des Polynoms $\mathbf{R}_{v}^{A}$ bekannt, so können die Koeffizienten des gesuchten Polynoms aus Gleichung (38), wie im Folgenden gezeigt, analytisch berechnet werden. Steht nur die Matrix $\mathbf{R}_{v}$ zur Verfügung, so wie es im Satz 3 der Fall ist, so muss vorerst die Adjunkte der Matrix $\mathbf{R}_{v}$, d. h. die Matrizen $\mathbf{N}_{c_{i}}, i=0, \ldots, \mu$, berechnet werden. Diese können z.B. mit Hilfe des Lemmas A.1 aus [11] berechnet werden. Eine Alternative zur Berechnung der Adjunkten $\mathbf{R}_{v}^{A}$ ist die numerische Berechnung von $\mathbf{S}_{v}^{\perp}$. Diese Vorgehensweise wird am Ende dieses Abschnittes vorgestellt.

Im Weiteren nehmen wir an, dass die Matrizen $\mathbf{N}_{c_{i}}$, $i=0, \ldots, \mu$, bekannt sind. Durch die Multiplikation der polynomialen Matrizen aus Gleichung (36) ergibt sich

$$
\mathbf{S}_{v}^{\perp} \mathbf{s}_{v}=\sum_{i=0}^{v} v^{i} \mathbf{S}_{c_{i}}^{\perp} \sum_{j=0}^{l} v^{j} \mathbf{s}_{c_{j}}=\mathbf{0}, \quad \forall v \in[\varepsilon, 1],
$$

$\operatorname{da} \operatorname{det}\left(\mathbf{R}_{v}\right) \neq 0, \forall v \in(\varepsilon, 1)$, und $v$ strikt positiv ist. Diese Gleichung ist offensichtlich erfüllt, wenn alle Koeffizienten des resultierenden polynomialen Vektors null sind. Dies ist äquivalent zu der Bedingung

$$
\left[\begin{array}{lll}
\mathbf{S}_{c_{v}}^{\perp} & \cdots & \mathbf{S}_{c_{0}}^{\perp}
\end{array}\right]\left[\begin{array}{ccccc}
\mathbf{s}_{c_{l}} & \cdots & \mathbf{s}_{c_{0}} & \cdots & \mathbf{0} \\
\vdots & \ddots & \ddots & \ddots & \vdots \\
\mathbf{0} & \cdots & \mathbf{s}_{c_{l}} & \cdots & \mathbf{s}_{c_{0}}
\end{array}\right]=\mathbf{0},
$$

wobei die konstante Matrix

$$
\mathbf{S}:=\left[\begin{array}{lll}
\mathbf{S}_{c_{v}}^{\perp} & \cdots & \mathbf{S}_{c_{0}}^{\perp}
\end{array}\right] \in \mathbb{R}^{(n-1) \times n(v+1)}
$$

die Koeffizienten des gesuchten Polynoms gruppiert, und die konstante Matrix

$$
\mathbf{S}_{\mathrm{G}}:=\left[\begin{array}{ccccc}
\mathbf{s}_{\mathcal{c}_{l}} & \cdots & \mathbf{s}_{\mathcal{c}_{0}} & \cdots & \mathbf{0} \\
\vdots & \ddots & \ddots & \ddots & \vdots \\
\mathbf{0} & \cdots & \mathbf{s}_{\mathcal{G}_{1}} & \cdots & \mathbf{s}_{c_{0}}
\end{array}\right] \in \mathbb{R}^{n(v+1) \times(l+1+v)},
$$

mit $r:=\operatorname{Rang}\left(\mathbf{S}_{\mathrm{G}}\right)$, die Koeffizienten der bekannten Umschaltfunktion gruppiert. Die Bestimmung der Matrix $\mathbf{S}$ kann aus der Singulärwertzerlegung der Matrix $\mathbf{S}_{\mathrm{G}}$ gewonnen werden, d. h. aus $\mathbf{S}_{\mathrm{G}}^{\perp}=\mathbf{H}_{1} \mathbf{U}_{2}^{\top}$, wobei die unitäre Matrix $\mathbf{U}_{2} \in \mathbb{R}^{n(v+1) \times(v+1) n-r}$ aus

$$
\mathbf{S}_{\mathrm{G}}=\left[\begin{array}{ll}
\mathbf{U}_{1} & \mathbf{U}_{2}
\end{array}\right]\left[\begin{array}{cc}
\Sigma\left(\mathbf{S}_{\mathrm{G}}\right) & \mathbf{0} \\
\mathbf{0} & \mathbf{0}
\end{array}\right]\left[\begin{array}{l}
\mathbf{V}_{1}^{\top} \\
\mathbf{V}_{2}^{\top}
\end{array}\right]
$$

entnommen wird. Eine beliebige nichtsinguläre Matrix $\mathbf{H}_{1} \in \mathbb{R}^{(v+1) n-r \times(v+1) n-r}$ wird so gewählt, dass für die resultierende Matrix $\mathbf{S}_{\mathrm{G}}^{\perp} \in \mathbb{R}^{n(v+1)-r \times n(v+1)}$ gilt. Somit ergibt sich $\mathbf{S}_{\mathrm{G}}^{\perp} \mathbf{S}_{\mathrm{G}}=\mathbf{H}_{1} \mathbf{U}_{2}^{\top} \mathbf{S}_{\mathrm{G}}=\mathbf{H}_{1} \mathbf{U}_{2}^{\top} \mathbf{U}_{1} \Sigma\left(\mathbf{S}_{\mathrm{G}}\right) \mathbf{V}_{1}^{\top}=\mathbf{0}$, da $\mathbf{U}_{2}^{\top} \mathbf{U}_{1}=\mathbf{0}$. Dabei gilt noch

$$
\begin{aligned}
& n(v+1)-r=\min \left\{\operatorname{Rang}\left(\mathbf{H}_{1}\right), \operatorname{Rang}\left(\mathbf{U}_{2}^{\top}\right)\right\} \\
& \geq \operatorname{Rang}\left(\mathbf{S}_{\mathrm{G}}^{\perp}\right) \\
& \geq \operatorname{Rang}\left(\mathbf{H}_{1}\right)+\operatorname{Rang}\left(\mathbf{U}_{2}^{\top}\right)-n(v+1)+r \\
& \quad=n(v+1)-r,
\end{aligned}
$$

d. h. $\operatorname{Rang}\left(\mathbf{S}_{\mathrm{G}}^{\perp}\right)=n(v+1)-r$. Die gesuchte Matrix $\mathbf{S}$ ist schließlich gegeben durch

$$
\mathbf{S}=\mathbf{H}_{2} \mathbf{S}_{\mathrm{G}}^{\perp}, \quad \mathbf{H}_{2} \in \mathbb{R}^{(n-1) \times n(v+1)-r},
$$

wobei die Matrix $\mathbf{H}_{2}$ eine beliebige Matrix mit $\operatorname{Rang}\left(\mathbf{H}_{2}\right)=$ $\min \{n-1,(v+1) n-r\}$ ist. Es gilt folglich

$$
\begin{aligned}
\min \{n-1,(v+1) n-r\} \\
=\min \left\{\operatorname{Rang}\left(\mathbf{H}_{2}\right), \operatorname{Rang}\left(\mathbf{S}_{\mathrm{G}}^{\perp}\right)\right\} \\
\geq \operatorname{Rang}(\mathbf{S}) \\
\geq \operatorname{Rang}\left(\mathbf{H}_{2}\right)+\operatorname{Rang}\left(\mathbf{S}_{\mathrm{G}}^{\perp}\right)-n(v+1)+r \\
=\min \{n-1,(v+1) n-r\},
\end{aligned}
$$

d. h. $\operatorname{Rang}(\mathbf{S})=\min \{n-1,(v+1) n-r\}$. Für das gesuchte Polynom ergibt sich

$$
\mathbf{S}_{v}^{\perp}=\left[\begin{array}{lll}
\mathbf{S}_{c_{v}}^{\perp} & \cdots & \mathbf{S}_{c_{0}}^{\perp}
\end{array}\right]\left[\begin{array}{c}
v^{v} \mathbf{I}_{n} \\
\vdots \\
\mathbf{I}_{n}
\end{array}\right]=\mathbf{S}\left[\begin{array}{c}
v^{v} \mathbf{I}_{n} \\
\vdots \\
\mathbf{I}_{n}
\end{array}\right],
$$

mit $\mathbf{S} \in \mathbb{R}^{(n-1) \times n(v+1)}$, wobei

$$
\operatorname{Rang}\left(\mathbf{S}_{v}^{\perp}\right) \leq \min \{n-1,(v+1) n-r\}
$$

Eine analytische Form des gesuchten Polynoms steht somit zur Verfügung. Diese Methode stellt jedoch nicht sicher, dass die Bedingung (37) erfüllt ist. Dies wäre der Fall, wenn $\operatorname{Rang}\left(\mathbf{S}_{v}^{\perp}\right)=n-1$ wäre. Die Bedingung aus Gleichung (37) kann aber in eine LMI transformiert werden. 
Dies wird im Folgenden noch gezeigt. Man bildet erstens die Polynommultiplikation

$$
\mathbf{M}_{v}:=\mathbf{S}_{v}^{\perp}\left(\mathbf{S}_{v}^{\perp}\right)^{\top}=\sum_{j=0}^{2 v} \mathbf{M}_{c_{j}} v^{j},
$$

wobei

$$
\mathbf{M}_{c_{j}}:=\sum_{\substack{i=0 \\ j-v \leq i \leq v}}^{j} \mathbf{S}_{c_{i}}^{\perp}\left(\mathbf{S}_{c_{j-i}}^{\perp}\right)^{\top}, \quad 0 \leq j \leq 2 v .
$$

Gleichung (37) ist somit äquivalent zu

$$
\mathbf{M}_{v}=\sum_{j=0}^{2 v} \mathbf{M}_{c_{j}} v^{j}>\mathbf{0}, \quad \forall v \in[\varepsilon, 1] .
$$

Dies ist für $\tilde{v}:=(1 / \alpha) v-\beta / \alpha$, mit $\alpha:=(1-\varepsilon) / 2$ und $\beta:=$ $(1+\varepsilon) / 2$, weiterhin äquivalent zu

$$
\tilde{\mathbf{M}}_{\tilde{v}}=\sum_{j=0}^{2 v} \tilde{\mathbf{M}}_{c_{j}} \tilde{v}^{j}>\mathbf{0}, \quad \forall \tilde{v} \in[-1,1],
$$

mit

$$
\tilde{\mathbf{M}}_{c_{j}}=\sum_{i=j}^{2 v}\left(\begin{array}{l}
i \\
j
\end{array}\right) \alpha^{j} \beta^{i-j} \mathbf{M}_{c_{i}}, \quad 0 \leq j \leq 2 v .
$$

Zweitens wird die Matrix $\tilde{\mathbf{M}}_{\tilde{v}}$ aus Gleichung (41) in der Form

$$
\tilde{\mathbf{M}}_{\tilde{v}}=\left(\tilde{\mathbf{v}}^{[v+1]} \otimes \mathbf{I}_{n}\right)^{\top} \tilde{\mathbf{M}}_{\Sigma}\left(\tilde{\mathbf{v}}^{[v+1]} \otimes \mathbf{I}_{n}\right)
$$

mit

$$
\tilde{\mathbf{M}}_{\Sigma}:=\frac{1}{2}\left[\begin{array}{ccccc}
2 \tilde{\mathbf{M}}_{c_{0}} & \tilde{\mathbf{M}}_{c_{1}} & \mathbf{0} & \ldots & \mathbf{0} \\
\tilde{\mathbf{M}}_{c_{1}} & 2 \tilde{\mathbf{M}}_{c_{2}} & \tilde{\mathbf{M}}_{c_{3}} & \ddots & \vdots \\
\mathbf{0} & \tilde{\mathbf{M}}_{c_{3}} & 2 \tilde{\mathbf{M}}_{c_{4}} & \ddots & \mathbf{0} \\
\vdots & \ddots & \ddots & \ddots & \tilde{\mathbf{M}}_{\mathcal{c}_{v-1}} \\
\mathbf{0} & \ldots & \mathbf{0} & \tilde{\mathbf{M}}_{c_{2 v-1}} & 2 \tilde{\mathbf{M}}_{c_{2 v}}
\end{array}\right]
$$

geschrieben. Die Matrix $\tilde{\mathbf{M}}_{\tilde{v}}$ ist positiv definit für jedes $\tilde{v} \epsilon$ $[-1,1]$ dann und nur dann, wenn zwei Matrizen, $\mathbf{D} \in \mathbb{P}^{n v}$ und $\mathbf{G} \in$ Skew $^{n v}$ existieren, sodass

$$
\begin{gathered}
\tilde{\mathbf{M}}_{\tilde{v}}>\left[\begin{array}{l}
\hat{\mathbf{J}}_{v} \otimes \mathbf{I}_{n} \\
\check{\mathbf{J}}_{v} \otimes \mathbf{I}_{n}
\end{array}\right]^{\top}\left[\begin{array}{cc}
-\mathbf{D} & \mathbf{G} \\
\mathbf{G}^{\top} & \mathbf{D}
\end{array}\right]\left[\begin{array}{l}
\hat{\mathbf{J}}_{v} \otimes \mathbf{I}_{n} \\
\check{\mathbf{J}}_{v} \otimes \mathbf{I}_{n}
\end{array}\right], \\
\hat{\mathbf{J}}_{v}:=\left[\begin{array}{ll}
\mathbf{I}_{v} & \mathbf{0}_{v, 1}
\end{array}\right], \quad \check{\mathbf{J}}_{v}:=\left[\begin{array}{ll}
\mathbf{0}_{v, 1} & \mathbf{I}_{v}
\end{array}\right],
\end{gathered}
$$

gilt. ${ }^{18}$ Die Bedingung aus Gleichung (42) ist eine parameterunabhängige LMI Bedingung, welche numerisch effizient überprüft werden kann.

18 Vgl. [20]. Diese Äquivalenzbedingung beruht auf eine verallgemeinerte $S$-Prozedur, welche in [10] eingeführt wurde. $\mathbf{A} \otimes \mathbf{B}$ bezeichnet dabei das Kronecker-Produkt.
Zusammenfassend ergibt sich die notwendige Verstärkung $\kappa_{v}=0.5 \mu_{v}$ aus Gleichung (30), mit dem Polynom $\mathbf{S}_{v}^{\perp}$ aus Gleichung (38), dessen Koeffizienten in der Matrix $\mathbf{S}$ aus Gleichung (39) gruppiert sind. Die Matrix $\mathbf{S}$ kann aus der Singulärwertzerlegung der Matrix $\mathbf{S}_{\mathrm{G}}$ berechnet werden. Anschließend muss Bedingung (37) überprüft werden.

Eine Alternative zur obigen Berechnung ist eine Berechnung während des Ausregelvorgangs. Für jedes $v^{*} \epsilon$ $[\varepsilon, 1]$ kann die Matrix $\mathbf{S}_{v^{*}}^{\perp}$ aus der Singulärwertzerlegung des Vektors $\mathbf{s}_{v^{*}}$ berechnet werden. Folglich hat die Matrix $\mathbf{S}_{v^{*}}^{\perp}$ die Form

$$
\mathbf{S}_{v^{*}}^{\perp}=\mathbf{H U}_{2_{v^{*}}}^{\top},
$$

mit einer beliebigen nichtsingulären Matrix $\mathbf{H} \in$ $\mathbb{R}^{(n-1) \times(n-1)}$ und Matrix $\mathbf{U}_{2_{v^{*}}} \in \mathbb{R}^{n \times(n-1)}$ aus der Singulärwertzerlegung ${ }^{19}$ von $\mathbf{s}_{v^{*}}$, d. h. ${ }^{20}$

$$
\mathbf{s}_{v^{*}}=\left[\begin{array}{ll}
\mathbf{U}_{v_{v^{*}}} & \mathbf{U}_{2_{v^{*}}}
\end{array}\right]\left[\begin{array}{ll}
\sigma\left(\mathbf{s}_{v^{*}}\right) & \mathbf{0}
\end{array}\right]^{\top} .
$$

Somit gilt $\mathbf{U}_{2_{v^{*}}}^{\top} \in \mathbb{R}^{n \times(n-1)}$ und $\operatorname{Rang}\left(\mathbf{U}_{2_{v^{*}}}^{\top}\right)=n-1 . .^{21}$ Die Matrix $\mathbf{S}_{v^{*}}^{\perp}$ hat dabei vollen Rang, d. h. $\operatorname{Rang}\left(\mathbf{S}_{v^{*}}^{\perp}\right)=n-1$. Dies ist ersichtlich aus

$$
\begin{aligned}
n-1= & \min \left\{\operatorname{Rang}(\mathbf{H}), \operatorname{Rang}\left(\mathbf{U}_{2_{v^{*}}}^{\top}\right)\right\} \\
\geq & \operatorname{Rang}\left(\mathbf{S}_{v^{*}}^{\perp}\right) \\
& \geq \operatorname{Rang}(\mathbf{H})+\operatorname{Rang}\left(\mathbf{U}_{2_{v^{*}}}^{\top}\right)-(n-1)=n-1 .
\end{aligned}
$$

\section{Regelungsentwurf}

Die in den Abschnitten 2 und 3 vorgestellten Regelgesetze können durch folgende Schritte entworfen werden:

1. Löse das Validierungsproblem (10)-(12).

2. Verwende das resultierende nichtsättigende Regelgesetz $u$ in der Form gegeben in Gleichung (8) und (13), mit den Parametern $d$ und $r$ aus Gleichung (14).

3. Alternativ, verwende das konvergenzoptimale Regelgesetz $u_{s}$ aus Gleichung (25) mit der Matrix $\mathbf{Q}_{v}$ aus Gleichung (13).

4. Alternativ, verwende den High-Gain-Regler $u_{\text {sat }}$ aus Gleichung (27) mit dem Parameter $\kappa_{v}=0.5 \mu_{v}$ und $\mu_{v}$ aus Gleichung (30), (38) und (40).

\section{Vgl. [16, Satz 2.1.1].}

20 Da die Blockmatrix $\left[\begin{array}{ll}\mathbf{U}_{1_{v^{*}}} & \mathbf{U}_{2_{v^{*}}}\end{array}\right]$ unitär ist, folgt, dass $\mathbf{U}_{2_{v^{*}}}^{\top} \mathbf{U}_{1_{v^{*}}}=$ $\mathbf{0}$ und, daher, dass $\mathbf{S}_{v^{*}}^{\perp} \mathbf{s}_{v^{*}}=\mathbf{H} \mathbf{U}_{2_{v^{*}}}^{\top} \mathbf{U}_{1_{v^{*}}} \sigma\left(\mathbf{s}_{v^{*}}\right)=\mathbf{0}$. Dabei ist $\sigma\left(\mathbf{s}_{v^{*}}\right)$ der Singulärwert von $\mathbf{s}_{v^{*}}$.

21 Die Matrix $\mathbf{U}_{v^{*}}=\left[\mathbf{U}_{1_{v^{*}}} \mathbf{U}_{2_{v^{*}}}\right] \in \mathbb{R}^{n \times n}$ ist unitär. Diese hat folglich $n$ unabhängige Spalten. Somit folgt, dass $\operatorname{Rang}\left(\mathbf{U}_{2 v^{*}}^{\top}\right)=n-1$. 
Das Validierungsproblem aus Punkt 1 beinhaltet keine Bedingungen bezüglich der Größe des Ellipsoids. Möchte man vermeiden, dass das Validierungsproblem ein zu kleines Ellipsoid erzeugt, so kann man weitere Bedingungen einführen. Beispielsweise kann man zusätzlich fordern, dass $\mathbf{P}_{1}^{-1} \prec \mathbf{C} \prec e \mathbf{I}_{n}$. Damit stellt man sicher, dass das Volumen des Ellipsoids $\mathcal{E}_{\mathrm{P}}(1)$ größer als das Volumen eines vorgegebenen Ellipsoids $\mathcal{E}(e):=\left\{\mathbf{x} \in \mathbb{R}^{n} \mid \mathbf{x}^{\top} e \mathbf{I x}<1\right\}$ ist. Erzielen kann man dies durch die zusätzlichen LMIs

$$
\left[\begin{array}{ll}
\mathbf{P}_{1} & \mathbf{I}_{n} \\
\mathbf{I}_{n} & \mathbf{C}
\end{array}\right]>\mathbf{0}, \quad \mathbf{C} \prec e \mathbf{I}_{n} .
$$

Auch die Wahl der Parameter $\mathrm{m}_{1}, \mathrm{~m}_{\mathrm{u}}$ und $\epsilon$ beeinflusst die Lösung des Validierungsproblems. Jedoch konnten keine großen Unterschiede in dem Ausregelverhalten bei den untersuchten Beispielen festgestellt werden.

Das Regelgesetz aus Punkt 2 wird im Allgemeinen nicht zu einem schnellen Ausregelverhalten führen. Aufgrund der Nichtkonservativität der Bedingungen des Validierungsproblems aus Punkt 1, stellt dieses Regelgesetz jedoch sicher, dass für die analysierte Strecke überhaupt ein Regelgesetz dieser Klasse existiert. Dieses kann auch als Startregler für einen Optimierungsalgorithmus verwendet werden. Das Regelgesetz aus Punkt 3 führt zu einem schnelleren Ausregelverhalten. Schließlich approximiert das Regelgesetz aus Punkt 4 die Regelgesetze aus Punkt 3.

\section{Beispiel: Fusionsreaktor}

Betrachtet wird folgende lineare Strecke mit einem instabilen und einem stabilen Eigenwert

$$
\dot{\mathbf{x}}=\left[\begin{array}{cc}
1 & 0 \\
0 & -0.5
\end{array}\right] \mathbf{x}+\left[\begin{array}{c}
1 \\
-0.5
\end{array}\right] u, \quad|u| \leq 1 .
$$

Das Modell beschreibt die wesentlichen physikalischen Eigenschaften von felderzeugenden Strömen, wobei die erzeugten Magnetfelder die Position des Plasmas in einem Fusionsreaktor halten. ${ }^{22}$ Bei dieser Strecke ist zu beachten, dass die Null-steuerbare Region $\mathbf{x} \in[-1,1] \times \mathbb{R}$ ist. Diese ist die Region im Zustandsraum, welche durch eine beschränkte Stellgröße $|u| \leq 1$ überhaupt ausregelbar ist. Diese Region ist beschränkt, da die Strecke einen instabilen Eigenwert bei $\lambda_{2}=1$ besitzt. ${ }^{23}$

22 Vgl. [6] und die Referenzen darin.

23 Vgl. [7, Proposition 2.2.1] und Gleichung (2.4.2) für die Analyse von Null-steuerbaren Regionen von Systemen mit reellen Eigenwerten.
Aus dem Validierungsproblem (10)-(12) mit $\mathrm{M}_{\mathrm{L}}=-1$ und $\mathrm{M}_{\mathrm{U}}=0$, sowie $\varepsilon=0.001$ resultieren die Matrizen

$$
\begin{aligned}
\mathbf{P}_{\mathrm{c}_{-1}} & =\left[\begin{array}{ll}
-0.0068 & -0.0331 \\
-0.0331 & -0.1660
\end{array}\right], \\
\mathbf{P}_{\mathrm{c}_{0}} & =\left[\begin{array}{ll}
11.0492 & 53.9821 \\
53.9821 & 271.1355
\end{array}\right] .
\end{aligned}
$$

Abbildung 1 zeigt mehrere Ellipsen im Zustandsraum. Die größte, $\partial \mathcal{E}_{\mathrm{p}}(1)(-)$, mit $\partial \mathcal{E}_{\mathrm{P}}(1)=\left\{\mathbf{x} \in \mathbb{R}^{n} \mid \mathbf{x}^{\top} \mathbf{P}_{1}^{-1} \mathbf{x}=1\right\}$, ist für $v=1$ aus dem Validierungsproblem (10)-(12) mit der zusätzlichen LMI aus Gleichung (43) und $e=3$ erzeugt. Diese kann skaliert werden, um einen beschränkten Regler zu erzielen. Für $r=1$ wird die Ellipse $\partial \mathcal{E}_{\mathrm{P}}^{\mathrm{d}}(1):=\{\mathbf{x} \in$ $\left.\mathbb{R}^{n} \mid \mathbf{x}^{\top} \mathbf{P}_{1}^{-1} \mathbf{x}=d\right\}(-$.$) mit d=\left(\mathbf{b}^{\top} \mathbf{P}_{\varepsilon}^{-1} \mathbf{b}\right)^{-1}=0.23$ erzielt. Die zweitgrößte Ellipse $\partial \varepsilon_{\mathrm{P}}^{\tilde{d}}(1):=\left\{\mathbf{x} \in \mathbb{R}^{n} \mid \mathbf{x}^{\top} \mathbf{P}_{1}^{-1} \mathbf{x}=\tilde{d}\right\}$ (- -) mit $\tilde{d}=\mathbf{x}_{0}^{\top} \mathbf{P}_{1}^{-1} \mathbf{x}_{0}=0.89$ wurde so gewählt, dass der Anfangszustand $\mathbf{x}_{0}=\left[\begin{array}{ll}0.58 & 2.8\end{array}\right]^{\top}$ auf deren Rand liegt. Die Existenz einer nichtsättigenden WSVR für diese skalierte Ellipse ist durch die Existenz eines Parameters $\tilde{r} \in[0.5,0.5098]$ sichergestellt, sodass Gleichung (14) erfüllt ist. Dies stellt aufgrund des Satzes 5 sicher, dass der konvergenzoptimale Regler und seine stetige Approximation ebenfalls die Strecke stabilisieren können.

Abbildung 2 zeigt die Simulationsergebnisse für vier verschiedene Regler, die nichtsättigende WSVR $u_{\mathrm{f}}$ aus Gleichungen (9), (13)-(14), der konvergenzoptimale Regler $u_{\mathrm{s}}$ aus Gleichung (25), der High-Gain-Regler $u_{\text {sat }}$ aus Gleichung (27) und der in [6, Abschnitt 7.2.2] vorgestellte nichtlineare Regler

$$
u_{\text {nonlin }}=\operatorname{sat}\left(-6 x_{1}-3 x_{2}\left(1-\left|x_{1}\right|\right)\right),
$$

der speziell für die Regelung linearer Strecken mit einem einzigen instabilen Pol und einer Stellgrößenbeschränkung entwickelt wurde. Der konvergenzoptimale und der High-Gain-Regler sind in den oberen zwei Abbildungen kaum zu unterscheiden. Es ist ersichtlich, dass der HighGain-Regler fast die gleiche Performance mit einem stetigen Regelgesetz erzielt.

\section{Beispiel: U-Boot}

Wir betrachten dazu eine Strecke dritter Ordnung mit zwei Eigenwerten bei Null und einem stabilen Eigenwert bei $\lambda_{3}=-0.005$

$$
\dot{\mathbf{x}}=\left[\begin{array}{ccc}
0 & 1 & 0 \\
0 & 0 & 1 \\
0 & 0 & -0.005
\end{array}\right] \mathbf{x}+\left[\begin{array}{c}
0 \\
0 \\
2.5 \cdot 10^{-5}
\end{array}\right] u, \quad|u| \leq 1 .
$$

Das Modell beschreibt die Dynamik der Tauchtiefe $x_{1}$ eines schwedischen U-Bootes der Firma Kockumation AB 


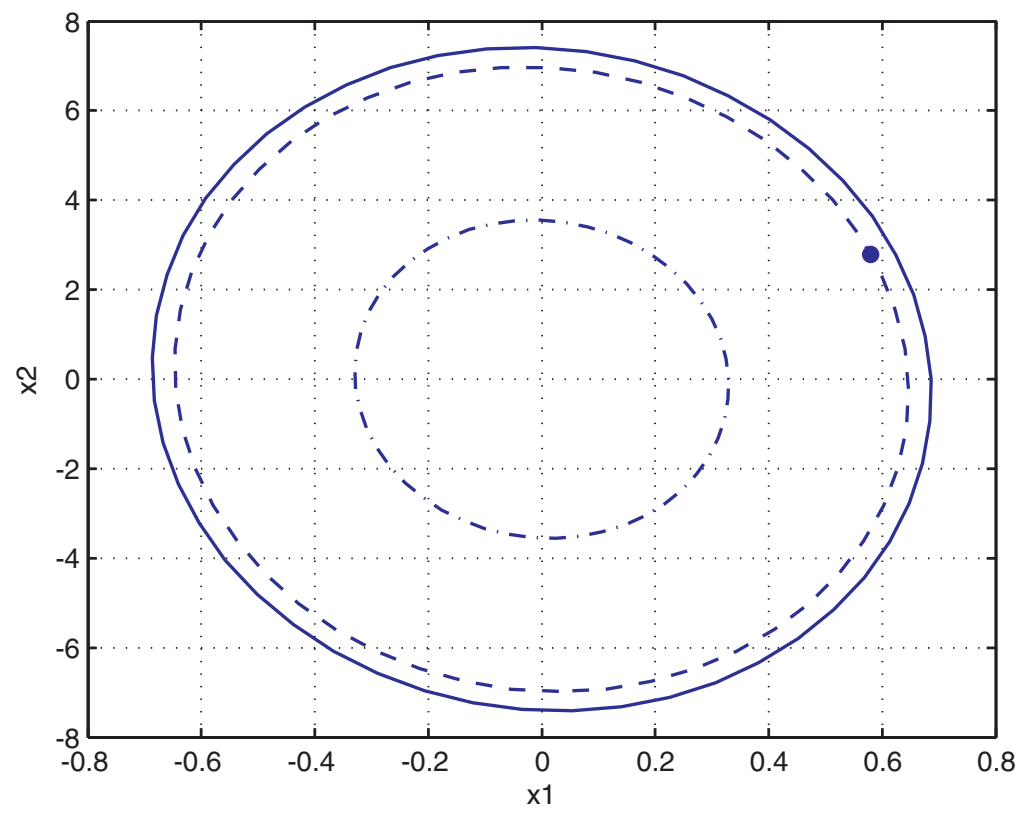

Abbildung 1: Anfangszustand $\mathrm{x}_{0}=\left[\begin{array}{ll}0.58 & 2.8\end{array}\right]^{\top}$ und die erzielten Ellipsoide für $v=1 \mathrm{im}$ Zustandsraum: aus der Lösung des Validierungsproblems $\partial \varepsilon_{P}(1)(-)$, das skalierte Ellipsoid für die Einhaltung der Stellgrößenbeschränkung, $\partial \varepsilon_{P}^{\mathrm{d}}(1)(-$.$) , und das$ skalierte Ellipsoid $\partial \varepsilon_{P}^{\tilde{d}}(1)$, auf dessen Rand die gewünschte Anfangsauslenkung liegt. und wurde aus [3] entnommen. Da die Strecke semi-stabil ist, ist die Null-steuerbare Region der gesamte Zustandsraum. Zu Vergleichszwecken wählen wir die Anfangsauslenkung $\mathbf{x}_{0}=\left[\begin{array}{lll}100 & 0 & 0\end{array}\right]^{\top}$. Verglichen werden die nichtsättigende WSVR, der konvergenzoptimale Regler und der High-Gain-Regler mit der zeitoptimalen Regelung aus [3, Abschnitt 4.2.5].

Das Validierungsproblem (10)-(12) mit $\mathrm{M}_{\mathrm{L}}=-1$, $\mathrm{M}_{\mathrm{U}}=0$ und $\varepsilon=0.01$ und der zusätzlichen Bedingung aus Gleichung (43) wird für verschiedene Werte von $e>0$ gelöst. Diese wurden so gewählt, dass keine Überschwingung bei $x_{1}$ entsteht. Die erzeugten Ellipsoide werden durch $\tilde{d}=\mathbf{x}_{0}^{\top} \mathbf{P}_{1}^{-1} \mathbf{x}_{0}$ skaliert, um die Anfangsauslenkung $\mathbf{x}_{0}$ simulieren zu können. Für diese Skalierung ist die Existenz einer nichtsättigenden WSVR durch ein $\tilde{r} \in\left[0.5, \tilde{r}_{\max }\right]$, mit $\tilde{r}_{\max }=\sqrt{1 /\left[d \cdot\left(\mathbf{b}^{\top} \mathbf{P}_{\varepsilon}^{-1} \mathbf{b}\right)\right]}$, sichergestellt, sodass Gleichung (14) erfüllt ist.

Tabelle 1 zeigt die Ergebnisse der Optimierung. Sie bestätigen die Wirkung der zusätzlichen LMI aus Gleichung (43), d. h. je höher $e$ ist, desto kleiner wird das Volumen des Ellipsoids $\varepsilon_{\mathrm{P}}(1)$, d. h. desto größer sind der Skalierungsfaktor $\tilde{d}$ und der Wert $\mathbf{b}^{\top} \mathbf{P}_{\varepsilon}^{-1} \mathbf{b}$. Somit sinkt $\tilde{r}_{\max }$ und für $e=0.01$ existiert keine nichtsättigende WSVR für das skalierte Ellipsoid, da $\tilde{r}_{\max }<0.5$ ist. Folglich ist die kontraktive Invarianz der Ellipsoide $\mathcal{E}_{\mathrm{P}}(v)$ im Falle der konvergenzoptimalen WSVR für $e=0.01$ nicht nachgewiesen. Eine alternative Methode, die kontraktive Invarianz der Ellipsoide im Falle der konvergenzoptimalen Regelung sicherzustellen, besteht in deren direkten Überprüfung, wie es beispielsweise in [8] für konvergenzoptimale Regelge-
Tabelle 1: Parameter $e>0$ aus der LMI aus Gleichung (43) und erzielter Wert von $\tilde{r}_{\max }$.

\begin{tabular}{lrrrrr}
\hline $\boldsymbol{e}$ & $\tilde{r}_{\min }$ & $\tilde{r}_{\max }$ & $\tilde{d}$ & $\mathbf{b}^{\top} \mathbf{P}_{\varepsilon}^{-1} \mathbf{b}$ & Symbol \\
\hline 0.0050 & 0.5 & 1.1430 & 29.78 & 0.0257 & $*$ \\
0.0071 & 0.5 & 0.5104 & 105.82 & 0.0363 & $\times$ \\
0.0100 & 0.5 & 0.2329 & 351.80 & 0.0524 & + \\
\hline
\end{tabular}

setze mit einer parameterunabhängigen Umschaltstrategie gezeigt wurde. Durch eine Erweiterung für parameterabhängige Umschaltstrategien (hier nicht gezeigt) ist es jedoch möglich, die kontraktive Invarianz für $e=0.01$ nachzuweisen.

Abbildung 3 zeigt die Ausregelverläufe für die analysierten Regler. Es ist ersichtlich, dass der festgelegte Parameter $e$ einen großen Einfluss auf den erzielten konvergenzoptimalen Regler hat und je höher $e$ ist, desto schneller ist das Ausregelverhalten.

\section{Zusammenfassung}

In dieser Arbeit wurden nichtlineare Regelgesetze für lineare Systeme mit einer Eingangsgröße und Stellgrößenbeschränkung untersucht. Ziel der Arbeit war es, nichtkonservative Existenzbedingungen für stabilisierende WSVR mit invers-polynomialen Selektionsstrategien herzuleiten und das resultierende Regelgesetz bezüglich der Konvergenzrate zu optimieren. Die Ergebnisse zeigen, dass das Regelgesetz, das die Konvergenzrate in jedem durch die Selektionsstrategie vorgegebenen Teil des Einzugsge- 

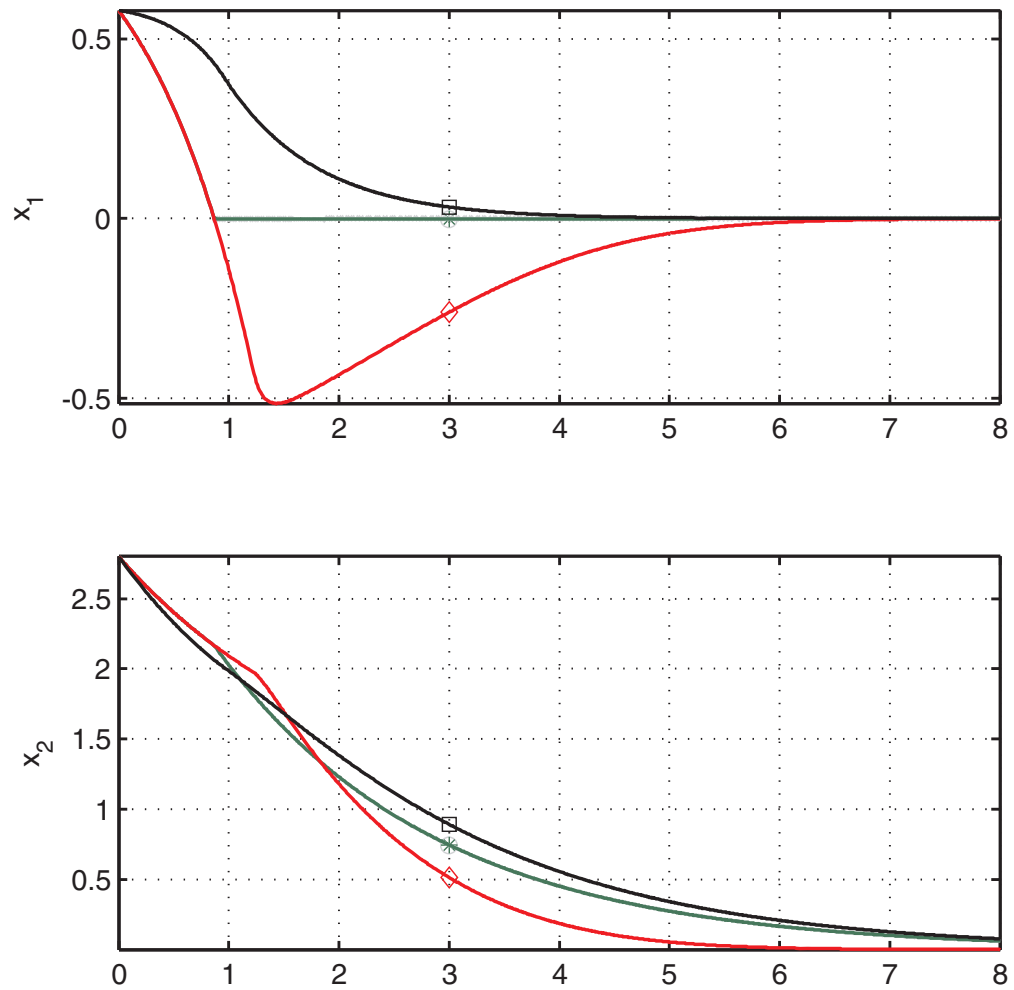

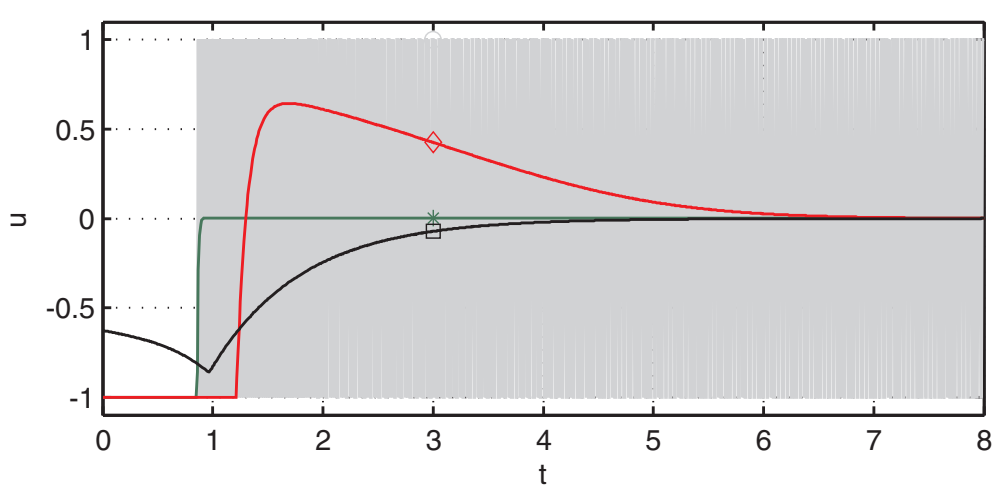

Abbildung 2: Simulation mit vier Reglern, die nichtsättigende WSVR $u_{\mathrm{f}}(\square)$, der konvergenzoptimale Regler $u_{s}(\bigcirc)$, der High-Gain-Regler $u_{\text {sat }}(*)$ und der nichtlinearer Regler $u_{\text {nonlin }}$ aus [6] $(\diamond)$. bietes maximiert, ein Bang-Bang-artiges Regelgesetz mit einer parameterabhängigen Umschaltstrategie ist.

Die hier vorgestellten Regelgesetze haben den Vorteil, dass sie nicht konservativ sind, da alle Entwurfsbedingungen sowohl hinreichend als auch notwendig sind. Sind beispielsweise diese Bedingungen nicht erfüllt, so existiert kein Regler dieser Klasse, der verschachtelte und kontraktiv invariante Gebiete im Zustandsraum erzeugt. Dies stellt auch einen wesentlichen Unterschied zu der polynomialen weich strukturvariablen Regelung aus [12] dar, wo nur hinreichende Stabilitätsbedingungen vorgestellt wurden. Darüber hinaus erzielt die Optimierung der Konvergenzrate einen Zweipunktregler mit einer einfachen parameterabhängigen Umschaltstrategie, welcher durch einen stetigen High-Gain-Regler ersetzt werden kann. Letzterer vermeidet die bekannten Nachteile des Zweipunktreglers, wie z.B. die ununterbrochene Aktivität des Zweipunktreglers aufgrund unvermeidbaren Rauschens. Die notwendige Verstärkung des High-Gain-Reglers ist darüber hinaus in einer sehr einfachen Form angegeben, welche abhängig von einer Basis des Nullraumes der Umschaltfunktion ist.

Das Beispiel aus Abschnitt 5 zeigt, dass die konvergenzoptimale WSVR mit invers-polynomialer Selektionsstrategie zu einer sehr schnellen Ausregelung führen kann. Das Beispiel aus Abschnitt 6 zeigt jedoch, dass der hier verwendete Ansatz, die Stabilität des Regelkreises mit der konvergenzoptimalen Regelung durch die Existenz 

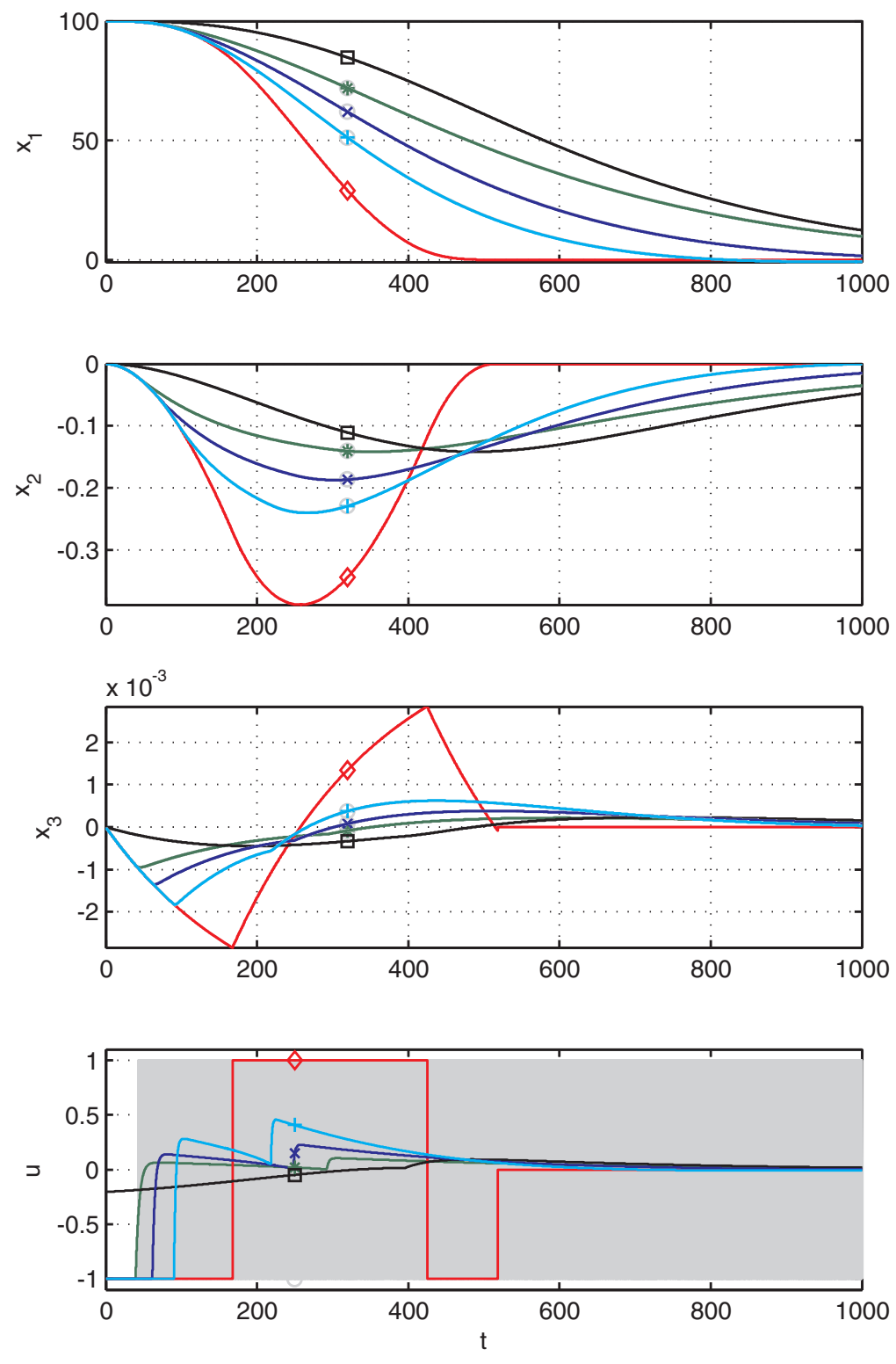

Abbildung 3: Simulation mit acht Reglern, die nichtsättigende WSVR $u_{\mathrm{f}}$ für $e=0.005$ und $\tilde{r}=\tilde{r}_{\text {max }}$ (口), die jeweiligen konvergenzoptimalen Regler $u_{s}$ $(\bigcirc)$, und High-Gain-Regler $u_{\text {sat }}$ für $e=0.005(*)$, $e=0.0071(\times)$ und $e=0.01(+)$, sowie der zeitoptimale Regler $u_{\text {opt }}(\diamond)$.

einer nichtsättigenden WSVR für das gleiche Einzugsgebiet der Ruhelage zu gewährleisten, einschränkend sein kann. Ein alternativer Ansatz, die kontraktive Invarianz des Einzugsgebietes im Falle der konvergenzoptimalen Regelung sicherzustellen, ist deren direkte Überprüfung, wie es beispielsweise in [8] für konvergenzoptimale Regelgesetze mit einer parameterunabhängigen Umschaltstrategie gezeigt wurde. Diesen Ansatz kann man für parameterabhängige Umschaltstrategien erweitern. Darüber hinaus wäre eine Übertragung der Regelungsmethode für Mehrgrößensysteme denkbar, da der vorgestellte Entwurf nicht auf Strecken in Steuerungsnormalform beschränkt ist. Dies bleibt Gegenstand weiterer Untersuchungen.

\section{Literatur}

1. J. Adamy und A. Flemming. Soft variable-structure controls: a survey. In: Automatica 40 (2004), S. 1821-1844.

2. J. Adamy. Implicit Lyapunov functions and isochrones of linear systems. In: IEEE Transactions on Automatic Control 50 (2005), Nr. 6, S. 874-879.

3. J. Adamy. Nichtlineare Systeme und Regelungen. 2. Aufl., Springer Vieweg, Berlin, Heidelberg, 2014.

4. D. S. Bernstein. Matrix mathematics: theory, facts, and formulas with application to linear systems theory. 2. Aufl., Princeton University Press, New Jersey, 2005.

5. F. Blanchini und S. Miani. Constrained stabilization of continuous-time linear systems. In: Systems \& Control Letters 28 (1996), Nr. 2, S. 95-102.

6. J.-Y. Favez. Enhancing the control of tokamaks via a continuous nonlinear control law. Diss., EPFL Lausanne, 2004. 
7. T. Hu und Z. Lin. On improving the performance with bounded continuous feedback laws. In: IEEE Transactions on Automatic Control 47 (2002), Nr. 9, S. 1570-1575.

8. T. Hu, Z. Lin, und Y. Shamash. On Maximizing the convergence rate for linear systems with input saturation. In: IEEE Transactions on Automatic Control 48 (2003), Nr. 7, S. 1249-1253.

9. T. Hu und Z. Lin. Control systems with actuator saturation. Analysis and design. Birkhäuser, Boston, 2001.

10. T. Iwasaki, G. Meinsma, und M. Fu. Generalized S-procedure and finite frequency KYP lemma. In: Mathematical Problems in Engineering 6 (2000), Nr. 2-3, S. 305-320.

11. T. Iwasaki, P. Tsiotras, und X. Zhang. State-feedback controller synthesis for parameter-dependent LTI systems. In: American Control Conference. Proceedings of the Vol. 1, S. 593-597, 8.10. Juni 2005.

12. B. Jasniewicz. Über weiche strukturvariable Regelung mittels impliziter Lyapunov-Funktionen - von der impliziten zur expliziten Regelung. In: Fortschr.-Ber. VDI Reihe 8, Nr. 1169, VDI Verlag, Düsseldorf, 2010.

13. H. Kiendl und G. Schneider. Synthese nichtlinearer regler für die regelstrecke const $/ s^{2}$ aufgrund ineinandergeschachtelter abgeschlossener gebiete beschränkter stellgröße. In: Regelungstechnik und Prozeß-Datenverarbeitung 20 (1972), S. 289296.

14. H. Lens, J. Adamy, und D. Domont-Yankulova. A fast nonlinear control method for linear systems with input saturation. In: Automatica 47 (2011), 857-860.

15. A. Röthig und J. Adamy. Entwurf konvergenzoptimaler weich strukturvariabler Regelungen für lineare Systeme mit Stellgrößenbeschränkung. In: at - Automatisierungstechnik 62 (2014), Nr. 12, S. 851-864.

16. R. E. Skelton, T. Iwasaki, und K. M. Grigoriadis. A unified algebraic approach to linear control design. Taylor \& Francis, New York, 1997.

17. J.-J. Slotine und W. Li. Applied nonlinear control. Prentice Hall, Englewood Cliffs, 1991.

18. S. Tarbouriech und M. Turner. Anti-windup design: an overview of some recent advances and open problems. In: IET Control Theory and Applications 3 (2007), Nr.1, S.1-19.

19. E.Zeidler. Springer-Taschenbuch der Mathematik. Begründet von I.N. Bronstein und K.A. Semendjaew. Weitergeführt von

Verfügbar unter

lediglich die vom Gesetz vorgesehenen Nutzungsrechte gemäß UrhG

\section{G. Grosche, V. Ziegler und D. Ziegler. Herausgegeben von}

E. Zeidler. 3. Aufl., Springer Spektrum, Wiesbaden, 2013.

20. X. Zhang. Parameter-dependent Lyapunov functions and stability analysis of linear parameter-dependent dynamical systems. Diss., School of Aerospace Engineering, Georgia Institute of Technology, 2003.

\section{Autoreninformationen}

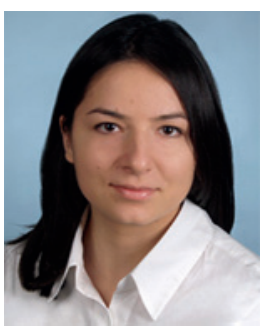

Dipl. Wirtsch.-Ing. Andreea Röthig Institut für Automatisierungstechnik und Mechatronik, Technische Universität Darmstadt, Landgraf-Georg-Str. 4, 64283 Darmstadt aroethig@rmr.tu-darmstadt.de

Dipl. Wirtsch.-Ing. Andreea Röthig promoviert am Fachgebiet Regelungsmethoden und Robotik der TU Darmstadt im Bereich nichtlineare Regelungen. Hauptarbeitsgebiete: Performance nichtlinearer Regelungen, weiche strukturvariable Regelungen, Systeme mit Stellgrößenbeschränkung.

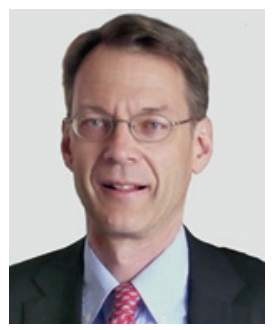

Prof. Dr.-Ing. Jürgen Adamy Institut für Automatisierungstechnik und Mechatronik, Technische Universität Darmstadt, Landgraf-Georg-Str. 4, 64283 Darmstadt adamy@rmr.tu-darmstadt.de

Prof. Dr.-Ing. Jürgen Adamy ist Leiter des Fachgebietes Regelungsmethoden und Robotik im Fachbereich Elektrotechnik und Informationstechnik der TU Darmstadt. Hauptarbeitsgebiete: Regelungsverfahren, Computational Intelligence, autonome mobile Roboter. 